\title{
Representation of the Category of Tangles by Kontsevich's Iterated Integral
}

\author{
Le Tu Quoc Thang, ${ }^{\star}$ Jun Murakami ${ }^{1}$ \\ Max-Planck-Institut für Mathematik, Gottfried-Claren-Straße 26, 53225 Bonn, Germany
}

Received: 12 August 1993/in revised form: 26 April 1994

\begin{abstract}
Applying Kontsevich's iterated integral for tangles, we get an isotopy invariant of tangles. We give a method to compute the integral of a tangle combinatorially from modified integrals of some simple tangles. We localize the integral by moving the end points of the tangle to an extreme configuration, and modify the integral so that it is convergent. By using a similar technique, we generalize Kontsevich's invariant to a framed tangle.
\end{abstract}

\section{Introduction}

After the Jones polynomial was discovered, many invariants of links are constructed. Almost all of them are coming from solutions of the quantum Yang-Baxter equation. On the other hand, Vassiliev [25] constructed a wide family of knot invariants. Let $\chi_{h}$ be a knot invariant coming from a solution $R(h)$ of the Yang-Baxter equation with a parameter $h$ such that $R(0)$ is the trivial solution. Then, $d^{k} \chi_{h} /\left.d h^{k}\right|_{h=0}$ is contained in Vassiliev's family of invariants. Hence, Vassiliev's invariants include many invariants, e.g. the Alexander, Jones, Homfly, Kauffman polynomials and their generalizations in $[1,17,20,22]$, etc. Kontsevich gives a universal construction of Vassiliev's invariant by using an "algebra of chord diagrams" and "iterated integrals." Let $V_{k}$ denote the space of Vassilev's invariants of degree less than $k+1$. By studying combinatorial properties of invariants in $V_{k}$, he constructs a module $\mathscr{A}_{0}^{(k)}$ spanned by chord diagrams on a circle with relations in Fig. 1 which correspond to the combinatorial relations for Vassiliev's invariants given in [7]. He shows that $V_{k} / V_{k-1}=\left(\mathscr{A}_{0}^{(k)}\right)^{*}$, the dual space of $\mathscr{A}_{0}^{(k)}$. Let $\mathscr{A}_{0}^{\prime}=\bigoplus_{k=0}^{\infty} A_{0}^{(k)}$. Then $\mathscr{A}_{0}^{\prime}$ has a graded algebra structure with a product coming from the connected sum of chord diagrams. The 4-term relation assures the well-definedness of the above product, i.e. the product does not depend on the positions of the strings we cut to produce the connected sum. Let $\mathscr{A}_{0}$ denote the formal completion of $\mathscr{A}_{0}^{\prime}$ with

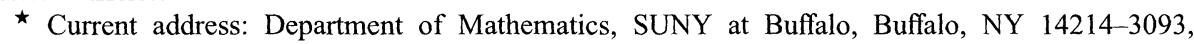
USA.

${ }^{1}$ On leave from Department of Mathematics, Osaka University, Toyonaka, Osaka 560, Japan
} 
a) 4-term relation

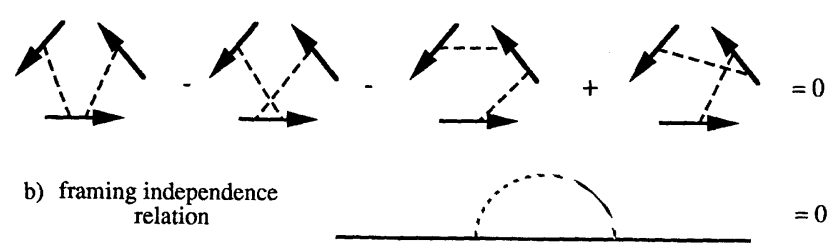

Fig. 1. 4-term relation and framing independence relation

respect to the grading. For every knot $K$, we can define an invariant $\widehat{Z}(K) \in \mathscr{A}_{0}$ by using an iterated integral. Let $\widehat{Z}^{(k)}(K) \in \mathscr{A}_{0}^{(k)}$ be the degree $k$ part of $\widehat{Z}(K)$. For $f \in \oplus_{k=0}^{\ell}\left(\mathscr{A}_{0}^{(k)}\right)^{*}, f\left(\widehat{Z}^{(k)}(K)\right) \in V_{\ell}$. On the other hand, for any $g \in V_{k}$, there is some $f \in \oplus_{k=0}^{\ell}\left(\mathscr{A}_{0}^{(k)}\right)^{*}$ such that $g(K)=f\left(\sum_{k=0}^{\ell} \widehat{Z}^{(k)}(K)\right)$ and $\widehat{Z}^{(k)}$ is universal in this sense.

Now we define $\mathscr{A}$, a linear span of chord diagrams with 4-term relations, but without the framing independence relation. There is a natural projection from $\mathscr{A}$ to $\mathscr{A}_{0}$. We extend $\widehat{Z}$ to a framed knot invariant $\widehat{Z}_{f}$.

The main purpose of this paper is to give a combinatorial description of Kontsevich's integral of knots, links and tangles. We will consider $\mathbf{R}^{3}$ as the product of $\mathbf{R}$ $\times \mathbf{C}$ with a fixed orientation. A point of $\mathbf{R}^{3}$ has coordinates $(t, z)$, let $z=x+i y$. A plane $\{t\} \times \mathbf{C} \subset \mathbf{R} \times \mathbf{C}$ is called horizontal. A tangle $T$ is a 1-dimensional compact oriented piece-wise smooth submanifold of $\mathbf{R}^{3}$ lying between two horizontal planes, called the top plane and the bottom plane of $\mathrm{T}$, such that every boundary point of $T$ is lying in the top and bottom plane. Two tangles $T$ and $T^{\prime}$ are called equivalent if there is an isotopy of $\mathbf{R}^{3}$ sending $T$ to $T^{\prime}$ and the top (resp. bottom) plane of $T$ to the top (resp. bottom) plane of $T^{\prime}$. Kontsevich's integral is generalized to an invariant of equivalence classes of tangles. We assume that a tangle $T$ is contained in $\mathbf{R} \times \mathbf{R} \subset \mathbf{R} \times \mathbf{C}$ except a neighborhood of each double point with respect to the projection $\mathbf{R} \times \mathbf{C} \rightarrow \mathbf{R} \times \mathbf{R}$. Especially the boundary points of $T$ are contained in $\mathbf{R} \times \mathbf{R}$. Let $r=\left(r_{1}, r_{2}, \ldots, r_{k}\right)\left(r_{j}= \pm 1\right)$ and $s=\left(s_{1}, s_{2}, \ldots, s_{\ell}\right)\left(s_{j}= \pm 1\right)$ such that $\#\left\{r_{j} \mid r_{j}=1\right\}-\#\left\{r_{j} \mid r_{j}=-1\right\}-\#\left\{s_{j} \mid s_{j}=1\right\}+\#\left\{s_{j} \mid s_{j}=-1\right\}=0$. Let $\mathscr{T}^{(r, s)}$ be a set of tangles such that the string ending at the $j^{\text {th }}$ point at the top is oriented upward (resp. downward) in the neighborhood of the end point if $r_{J}=1$ (resp. - 1) and the $j^{\text {th }}$ point at the bottom is oriented upward (resp. downward) if $s_{j}=1$ (resp. -1$)$. We generalize the integral $\widehat{Z}$ for tangles in the above sense. For a one-manifold $X$, let $\mathscr{A}(X)$ denote the space spanned by chord diagrams on $X$ with the 4-term relation. Every chord expressed by dashed lines in figures just means a pair of points on $X$ and nothing more. For $T \in \mathscr{T}^{(r, s)}$, we define an isotopy invariant $\widehat{Z}(T) \in \mathscr{A}(T)$ depending on the positions of the end points of $T$.

We introduce $q$-tangles, which is a generalization of tangles with non-associative words of two numbers 1 and -1 . A word $w$ with brackets is called a non-associative word of 1 and -1 if $w$ is equal to $1,-1$ or $\left(w_{1} w_{2}\right)$, where $w_{1}$ and $w_{2}$ are nonassociative words of 1 and -1. A support of $w$ is the sequence of 1 and -1 obtained from $w$ by removing all brackets. For example, there are two non-associative words $((11) 1)$ and $(1(11))$ with support $(1,1,1)$. Let $u$ and $v$ be non-associative words with support $r$ and $s$ respectively. The triple $(T, u, v)$ is called a $q$-tangle. As for 

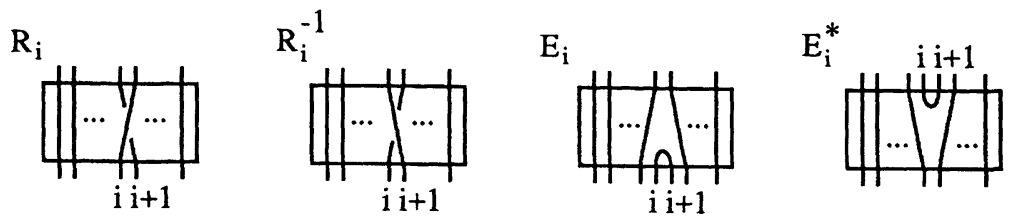

Fig. 2. Generator tangles

usual tangles, we can consider a category of q-tangles. For $T \in \mathscr{T}^{r, s}$ and $T^{\prime} \in \mathscr{T}^{r^{\prime}, s^{\prime}}$, the product of two q-tangles $(T, u, v)$ and $\left(T^{\prime}, u^{\prime}, v^{\prime}\right)$ is defined by

$$
(T, u, v)\left(T^{\prime}, u^{\prime}, v^{\prime}\right)=\left(T T^{\prime}, u, v^{\prime}\right),
$$

if $s=r^{\prime}$ and $v=u^{\prime}$, and $T T^{\prime}$ is the tangle obtained by putting $T$ above $T^{\prime}$. We show the following in Sect. 2.

Proposition 0.3. Every q-tangle is a product of tangles of the following types. The first type is a positive crossing $\left(R_{j}, u, u\right)$, where $u$ contains $\left(r_{j} r_{j+1}\right)$. The second type is a negative crossing $\left(R_{j}^{-1}, u, u\right)$, where $u$ contains $\left(r_{j} r_{j+1}\right)$. The next type is $\left(E_{J}, u, v\right)$, where $v$ contains $\left(r_{j} r_{J+1}\right)$ and $u$ is obtained from $v$ by removing $\left(r_{J} r_{l+1}\right)$ and its dual type $\left(E_{j}^{*}, u, v\right)$, where $u$ contains $\left(r_{j} r_{j+1}\right)$ and $v$ is obtained from $u$ by removing $\left(r_{j} r_{j+1}\right)$. The last types are $(I, u, v)$ and $(I, v, u)$, where $u$ is any non-associative word and $v$ is obtained from $u$ by replacing a subword of $u$ of the form $\left(u_{1}\left(u_{2} u_{3}\right)\right)$ by $\left(\left(u_{1} u_{2}\right) u_{3}\right)$. In the above, $R_{j}, R_{j}^{-1}, E_{j}, E_{j}^{*}$ are tangles as in Fig. 2, and $I$ is a trivial tangle.

By this proposition, we can compute the integral for any q-tangle from the modified integrals of the q-tangles in the above proposition. Let $\Omega_{p q}$ denote the horizontal chord connecting the $p^{\text {th }}$ and $q^{\text {th }}$ strings of the trivial tangle, and $\Omega^{(r)} \ldots \Omega^{(1)}$ denote the chord diagram with chords $\Omega^{(r)}, \ldots, \Omega^{(1)}$ from top to bottom in turns. We use $e^{X}$ for the formal power series $\sum_{k=0}^{\infty} X^{k} / k$ !. Let $P_{J j+1}$ denote the diagram presenting the permutation at $i^{\text {th }}$ and $j+1^{\text {th }}$ strings as in Fig. 3 .

Theorem 0.4. We define $\widehat{Z}_{f}$ for generator tangles as follows:

$$
\begin{aligned}
& \widehat{Z}_{f}\left(R_{j}, u, u\right)=e^{r_{J} r_{J+1} \Omega_{J j+1} / 2} P_{j J+1}, \\
& \widehat{Z}_{f}\left(R_{j}^{-1}, u, u\right)=e^{-r_{j} r_{j+1} \Omega_{\jmath}+1 / 2} P_{j J+1} \text {, } \\
& \widehat{Z}_{f}\left(E_{j}^{*}, u, v\right)=E_{j}^{*}, \\
& \widehat{Z}_{f}\left(E_{j}, u, v\right)=\gamma_{f}^{-1} \cdot E_{j}, \\
& \widehat{Z}_{f}(I, u, v)=\phi\left(\sum_{\substack{10 \leqq p \leqq I_{1}-1 \\
I_{1} \leqq q \leqq I_{2}-1}} r_{p} r_{q} \Omega_{p q}, \sum_{\substack{I_{1} \leqq p \leqq / 2-1 \\
J_{2} \leqq q \leqq J_{3}-1}} r_{p} r_{q} \Omega_{p q}\right), \\
& \widehat{Z}_{f}(I, v, u)=\phi\left(\sum_{\substack{J_{1} \leqq p \leqq J_{2}-1 \\
J_{2} \leqq q \leqq /_{3}-1}} r_{p} r_{q} \Omega_{p q}, \sum_{\substack{I_{0} \leqq p \leqq J_{1}-1 \\
J_{1} \leqq q \leqq J_{2}-1}} r_{p} r_{q} \Omega_{p q}\right) \text {, }
\end{aligned}
$$


where $\gamma_{f}=Z_{f}(U)$ is given by (5.7) for a diagram $U$ in Fig. 5, and the action of $\gamma_{f}$ is induced by the connected sum at the distinguished strings. For the last two formulas, $\phi$ is given in (3.7) and (3.21), $v$ is obtained from $u$ by replacing a subword for $u$ of the form $\left(u_{1}\left(u_{2} u_{3}\right)\right)$ by $\left(\left(u_{1} u_{2}\right), u_{3}\right)$, the support of $u_{1}, u_{2}$ and $u_{3}$ are $\left(r_{j_{0}}, \ldots, r_{j_{1}-1}\right),\left(r_{j_{1}}, \ldots, r_{j_{2}-1}\right)$, and $\left(r_{j_{2}}, \ldots, r_{j_{3}-1}\right)$ respectively. In the right-hand sides of the above, $E_{j}^{*}$ and $E_{j}$ represent the chord diagrams without any chords on $E_{j}^{*}$ and $E_{j}$ respectively.

Then $\widehat{Z}_{f}$ defines a representation of q-framed-tangles. Especially, for a knot $K$, the image $\widehat{Z}(K)$ of $\widehat{Z}_{f}(K)$ by the projection $\mathscr{A} \rightarrow \mathscr{A}_{0}$ in [5] is equal to Kontsevich's integral invariant.

Remark. 0.6. For a sequence $r$, let $w_{r}=\left(\left(\left(\ldots\left(r_{1} r_{2}\right) \ldots\right) r_{|r|-1}\right) r_{|r|}\right)$. Then, for a tangle $T \in \mathscr{T}^{(r, s)}, \widehat{Z}\left(\left(T, w_{r}, w_{s}\right)\right)$ and $\widehat{Z}_{f}\left(\left(T, w_{r}, w_{s}\right)\right)$ are invariants of a tangle and a framed tangle respectively. They also give representations of the categories of tangles and framed tangles respectively.

Knots can be expressed as a product of q-tangles in Proposition 0.3 as in the following example.

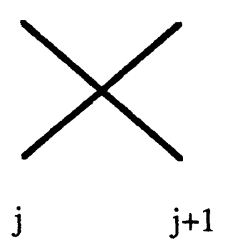

Fig. 3. $P_{J j+1}$

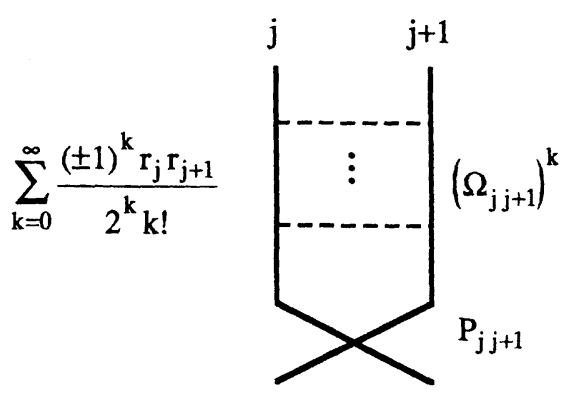

Fig. 4. $e^{ \pm \prime \prime r_{j+1} \Omega_{J J+1} / 2} P_{J J+1}$

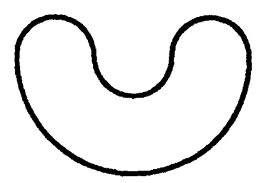

Fig. 5. Diagram U 


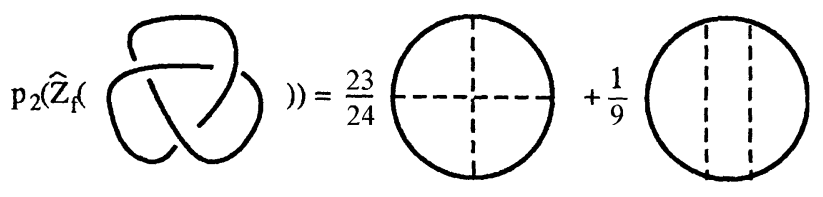

Fig. 6. The second degree part of $\widehat{Z}_{f}$ (trefoil)

Example 0.7. Let $K$ be the trefoil knot and $U$ be the trivial knot diagram as in Fig. 4. Then they are $(0,0)$-tangles

$$
\left(E_{1}, u_{0}, u_{1}\right)\left(E_{2}, u_{1}, u_{2}\right)\left(I, u_{2}, u_{3}\right)\left(R_{1}, u_{3}, u_{3}\right)^{3}\left(I, u_{3}, u_{2}\right)\left(E_{2}^{*}, u_{2}, u_{1}\right)\left(E_{1}^{*}, u_{1}, u_{0}\right),
$$

and

$$
\left(E_{1}, v_{0}, v_{1}\right)\left(E_{2}, v_{1}, v_{2}\right)\left(I, v_{2}, v_{3}\right)\left(E_{1}^{*}, v_{3}, v_{1}\right)\left(E_{1}^{*}, v_{1}, v_{0}\right)
$$

where $u_{0}=v_{0}=$ the null-word, $u_{1}=v_{1}=(1-1), u_{2}=((1(1-1))-1), u_{3}=$ $(((11)-1)-1), v_{2}=((1(-11))-1)$ and $v_{3}=(((1-1) 1)-1)$. Hence

$$
\begin{aligned}
\widehat{Z}_{f}(K) & =\gamma_{f}^{-1} \cdot E_{1} \gamma_{f}^{-1} \cdot E_{2} \phi\left(\Omega_{12},-\Omega_{23}\right) e^{3 \Omega_{12} / 2} P_{12} \phi\left(-\Omega_{23}, \Omega_{12}\right) E_{2}^{*} E_{1}^{*} \\
& =\gamma_{f}^{-2} \cdot E_{1} \cdot E_{2} \phi\left(\Omega_{12},-\Omega_{23}\right) e^{3 \Omega_{12} / 2} P_{12} \phi\left(-\Omega_{23}, \Omega_{12}\right) E_{2}^{*} E_{1}^{*}
\end{aligned}
$$

where $\phi\left(\Omega_{12}, \Omega_{23}\right)=1+\frac{\zeta(2)}{4 \pi^{2}}\left(\Omega_{12} \Omega_{23}-\Omega_{23} \Omega_{12}\right)+\cdots$ as in (3.10), $\gamma_{f}=E_{1} E_{2}$ $\phi\left(\Omega_{23}, \Omega_{12}\right) E_{1}^{*} E_{1}^{*}=1+\frac{1}{24} E_{1} E_{2}\left(\Omega_{12} \Omega_{23}-\Omega_{23} \Omega_{12}\right) E_{1}^{*} E_{1}^{*}+\cdots \quad$ by $(5.7)$ and so $\gamma_{f}{ }^{-2}=1-\frac{1}{12} E_{1} E_{2}\left(\Omega_{12} \Omega_{23}-\Omega_{23} \Omega_{12}\right) E_{1}^{*} E_{1}^{*}+\cdots$. For example, the degree two part of $\widehat{Z}_{f}(K)$ is $E_{1} E_{2}\left(\frac{23}{24} \Omega_{12}^{2}+\frac{1}{6} \Omega_{23}^{2}\right) P_{12} E_{2}^{*} E_{1}^{*}$.

Let $K_{1} \# K_{2}$ be the connected sum of two knots $K_{1}$ and $K_{2}$. Then, from the definition (5.8) of $\widehat{Z}_{f}$, we have

$$
\widehat{Z}_{f}\left(K_{1} \# K_{2}\right)=\gamma_{f} \cdot \widehat{Z}_{f}\left(K_{1}\right) \cdot \widehat{Z}_{f}\left(K_{2}\right) .
$$

For a framed link $L$ with a component $\ell$, let $\mathscr{D}_{\ell}(L)$ be the framed link obtained from $L$ whose component $\ell$ is doubled. Then

$$
\widehat{Z}_{f}\left(\mathscr{D}_{\ell}(L)\right)=\Delta_{\ell}\left(\widehat{Z}_{f}(L)\right) \in \mathscr{A}\left(D_{\ell}(L)\right)
$$

where $\Delta_{\ell}(\mathscr{D})$ for a chord diagram $\mathscr{D} \in \mathscr{A}(L)$ is defined as follows. To get $\Delta_{\ell}(\mathscr{D})$, we replace each chord $\Omega_{p q}$ with end points $p$ and $q$ by $\sum_{l, j=1}^{2} \Omega_{p_{l} q_{J}}$, where $p_{1}, p_{2}$ (resp. $q_{1}, q_{2}$ ) are points on the doubled string corresponding to $p$ (resp. $q$ ) if $p$ and $q$ are on the component $\ell, \sum_{l=1}^{2} \Omega_{p_{l} q}$ or $\sum_{i=1}^{2} \Omega_{p q_{l}}$ if $p$ or $q$ is on $\ell$, or $\Omega_{p q}$ if neither $p$ nor $q$ is on $\ell$.

The modified integral for trivial q-tangles is not necessarily trivial as in (0.5). However, this integral satisfies the properties of the associator in the theory of quasiHopf algebras in $[8,9]$. Moreover, the above $\Delta$ corresponds to the coproduct. With our $\phi$ in (0.5) and $\Delta$, we can impose a structure like a quasitriangular quasi-Hopf algebra on the modules of chord diagrams, as explained in Sect. 6.

In Sect. 1, we define Kontsevich's integral for tangles. In Sects. 2 and 3, we introduce pre-q-tangles and modified integrals for them. In Sect. 4, we show that the category of q-tangles is a quotient of the category of pre-q-tangles and the modified integral is factored by this quotient. We prove Theorem 0.4 from this fact. 
In Sect. 5, Kontsevich's integral is generalized for framed tangles. In Sect. 6, we introduce the quasi-Hopf algebra structure to our modified integrals.

After finishing our preparation of this paper, we got papers [6] and [3], which treat a similar subject from other points of view.

\section{Kontsevich's Integral for Tangles}

Let $X$ be a compact 1-dimensional oriented piece-wise smooth manifold with or without boundary. The components of $X$ are circles or segment lines. A chord diagram with support $X$ is a set of dashed chords with end points lying in the interior of $X$, regarded up to a diffeomorphism which preserves each component and the orientation of $X$. Connected components of $X$ are called strings, Wilson lines or Wilson loops. Let $\mathscr{A}(X)$ be the space spanned by chord diagrams with support in $X$ subject to the 4-term relations. Let $\mathscr{A}_{0}(X)$ be the space spanned by chord diagrams with support in $X$ subject to the 4-term relation and framing independence relation. The framing independence relation means that every chord diagram containing a part like Fig. 1 (b) is equal to zero. If $f: X \rightarrow X^{\prime}$ is a homeomorphism then there is an associate isomorphism between $\mathscr{A}(X)$ and $\mathscr{A}\left(X^{\prime}\right)$. If $X$ is a circle then we denote $\mathscr{A}(X)$ by $\mathscr{A}$. Let $\mathscr{D}_{1}$ and $\mathscr{D}_{2}$ be two chord diagrams in $\mathscr{A}$, each with a noted string. Remove an arc on each noted string which does not contain any vertex and then using two lines to combine the two strings into one single string. We get a chord diagram called the product (or connected sum) of $\mathscr{D}_{1}$ and $\mathscr{D}_{2}$ along the noted strings. It is proved in [5] that this operation does not depend on the location of the arcs removed. $\mathscr{A}$ has an algebra structure with this product. We denote by $\mathscr{A}_{0}$ the factored algebra of $\mathscr{A}$ by the framing independence relation. Using a connected sum and an evident isomorphism we can define an action of $\mathscr{A}$ on $\mathscr{A}(X)$ if the string to be acted on is indicated. The action is the connected sum with the indicated string. As in [5], it is easily proved that this action is well-defined. Similarly $\mathscr{A}_{0}$ acts on $\mathscr{A}_{0}(X)$.

Let $\mathscr{T}^{(r, s)}$ be a set of tangles as in the Introduction. Let $|r|$ and $|s|$ denote the numbers of elements in $r$ and $s$ respectively. For $T \in \mathscr{T}^{(r, s)}$, let $\mathscr{A}(T)$ denote the chord diagram algebra on $T$, i.e. the space spanned by chord diagrams with support in the 1-dimensional manifold corresponding to $T$ subject to the 4-term relation Fig. 1 (a), and let $\mathscr{A}_{0}(T)$ denote the quotient of $\mathscr{A}(T)$ by the framing independence relation Fig. 1 (b).

We generalize Kontsevich's iterated integral for knots to tangles. For a tangle $T$, we define an integral $Z(T) \in \mathscr{A}_{0}(T)$ as follows.

Definition 1.1. Let $Z(T)$ be the element of $A_{0}^{(k)}(T)$ defined by

$$
Z(T)=\sum_{n=0}^{\infty} \frac{1}{(2 \pi i)^{n}} \sum_{P / \sim}\left((-1)^{\# P_{\uparrow}} \int_{t_{1}<t_{2}<\cdots<t_{n}} \bigwedge_{j=1}^{n} \frac{d z_{j}\left(t_{j}\right)-d z_{j}^{\prime}\left(t_{j}\right)}{z_{j}\left(t_{j}\right)-z_{j}^{\prime}\left(t_{j}\right)}\right) T_{P} .
$$

In this equation, $P$ runs over all horizontal chord diagrams on $T$, where a horizontal chord diagram on $T$ means a chord diagram on $T$ with chords parallel to $\{0\} \times \mathbf{C}$ and two horizontal chord diagrams $\mathscr{D}_{1}$ and $\mathscr{D}_{2}$ are regarded to be equivalent if $\mathscr{D}_{2}$ can be obtained by moving the chords of $\mathscr{D}_{1}$ along with $T$ by keeping their order with respect to the level. The parameters $t_{1}, \ldots, t_{n}$ represent levels of the chords of $P$ and the integral is taken over all possible ranges $t_{1}, \ldots, t_{n}$ to represent all horizontal chord diagrams equivalent to $P$. The complex numbers $z_{j}\left(t_{j}\right)$ and $z_{j}^{\prime}\left(t_{j}\right)$ represent 


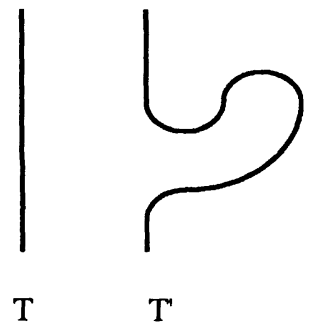

Fig. 7. $T$ and $T$ are identical except within a ball, in which they are as above

the horizontal coordinates of the end points of the $j^{\text {th }}$ chord. Note that the form does not depend on the choice of $z_{j}$ and $z_{j}^{\prime}$. \#P $P_{\uparrow}$ is the number of points $z_{j}$ and $z_{j}^{\prime}$ at which $L$ is oriented upwards, $T_{P}$ is the image of the chord diagram in $\mathscr{A}(T)$ naturally associated with $T$ and $P$.

Proposition 1.2. ([5, 16]) a) $Z(T)$ remains unchanged under isotopy which preserves every point of the bottom and the top planes and does not change the number of the maximal and minimal points of each string.

b) If $T^{\prime}$ differ from $T$ only in a neighborhood of a ball in which $T$ and $T^{\prime}$ look as Fig. 7, then

$$
Z\left(T^{\prime}\right)=\gamma \cdot Z(T)
$$

where $\gamma$ is the Kontsevich integral of the tangle $U$ in Fig. $5, \gamma$ belongs to $\mathscr{A}_{0}$ and the right side of this equality should be understood as the action of $\gamma$ on the string containing the part in Fig. 7.

Let $T$ be a tangle with $k$ numbered components. For $j=1, \ldots, k$ let $m_{j}$ be the number of the maximal points of the $j^{\text {th }}$ string. Let

$$
\widehat{Z}(T)=\left(\gamma^{-m_{1}} \otimes \cdots \otimes \gamma^{-m_{k}}\right) \cdot Z(T),
$$

here in the right-hand side, $\gamma^{-m_{J}}$ acts on the $j^{\text {th }}$ string.

Theorem 1.5 $\widehat{Z}(T)$ is an isotopy invariant of oriented tangles.

Proof. Using Proposition 1.2, one easily checks that $\widehat{Z}(T)$ is invariant under all the moves listed in Theorem 3.2 of [24]. Hence $\widehat{Z}(T)$ is an ambient isotopy invariant.

\section{Modified Integral for a Tangle}

Let $T \in \mathscr{T}^{(r, s)}$ and let $\varepsilon_{1}, \varepsilon_{2}, \ldots$ be positive real numbers. Let $S_{n}$ denote the permutation group of $n$ letters and let $\sigma$ (resp. $\tau$ ) be an element of $S_{|r|-1}$ (resp. $S_{|s|-1}$ ). Let $T_{\sigma, \tau, \varepsilon}$ be a tangle isotopic to $T$ such that the distance of the $j^{\text {th }}$ point and the $(j+1)^{\text {th }}$ point at the top is equal to $\varepsilon_{\sigma^{-1}(j)}$ and the distance of the $i^{\text {th }}$ point and the $(j+1)^{\text {th }}$ point at the bottom is equal to $\varepsilon_{\tau^{-1}(j)}$ as in Fig. 8. We use notation $\lim _{\varepsilon \rightarrow 0}$ for $\lim _{\varepsilon_{1} \rightarrow 0} \lim _{\varepsilon_{2} \rightarrow 0} \lim _{\varepsilon_{3} \rightarrow 0} \ldots$. The limit of the integral $\lim _{\varepsilon \rightarrow 0} Z\left(T_{\sigma, \tau, \varepsilon}\right)$ is not convergent. 


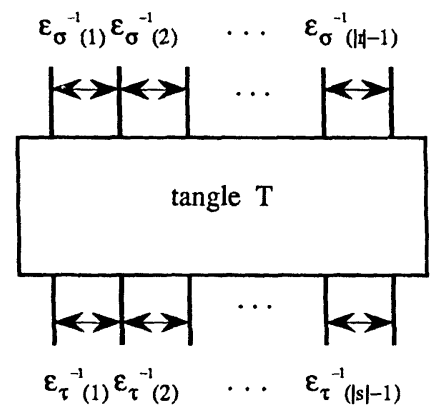

Fig. 8. $T_{\sigma, \tau, \approx}$

However, with some modification, we can get a part of this limit. For a positive real number $\varepsilon$, let $\varepsilon^{X}$ be a formal power series $\exp (X \log \varepsilon)$. Let

$$
\varepsilon_{\sigma, r}=\prod_{k=1}^{|r|-1} \varepsilon_{k}^{\Omega(\sigma, r, k)} \text {, and } \Omega(\sigma, r, k)=\sum_{\ell_{1} \leqq p \leqq \sigma(k) \sigma(k)+1 \leqq q \leqq \ell_{2}} \frac{r_{p} r_{q} \Omega_{p q}}{2 \pi i} \text {, }
$$

where $\ell_{1}$ and $\ell_{2}$ satisfy the following: $\sigma^{-1}(p)>k$ for all $\ell_{1} \leqq p<k$ and $\sigma^{-1}\left(\ell_{1}-1\right)<k$ if $\ell_{1}>1, \sigma^{-1}(q)>k$ for all $k+1 \leqq q<\ell_{2}$ and $\sigma^{-1}\left(\ell_{2}+1\right)<$ $k$ if $\ell_{2}<|r|$. All the terms $\varepsilon_{k}{ }^{\Omega(\sigma, r, k)}$ in (2.1) are mutually commutative and so $\varepsilon_{\sigma, r}$ is well-defined. We consider a limit of $Z\left(T_{\sigma, \tau, \varepsilon}\right)$ modified by $\varepsilon_{\sigma, r}$ and $\varepsilon_{\tau, s}$.

Theorem 2.2. $\lim _{\varepsilon \rightarrow 0} \varepsilon_{\sigma, r}^{-1} Z\left(T_{\sigma, \tau, \varepsilon}\right) \varepsilon_{\tau, s}$ is finite.

Proof of this theorem is given later.

Definition 2.3. For $T \in \mathscr{T}^{(r, s)}$ and $\sigma \in \mathscr{S}_{|r|-1}, \tau \in \mathscr{S}_{|s|-1}$, we call the triple $(T, \sigma, \tau)$ a pre-q-tangle. A category $C$ of pre-q-tangles is a category with objects $(r, \sigma)$ with $\sigma \in S_{|r|-1}$ and morphisms $(T, \sigma, \tau)$ from $(r, \sigma)$ to $(s, \tau)$. A product of two morphisms $\left(T_{1}, \sigma_{1}, \sigma_{2}\right)$ and $\left(T_{2}, \sigma_{2}, \sigma_{3}\right)$ with $T_{1} \in \mathscr{T}^{\left(r_{1}, r_{2}\right)}$ and $T_{2} \in \mathscr{T}^{\left(r_{2}, r_{3}\right)}$ is $\left(T_{1} T_{2}, \sigma_{1}, \sigma_{3}\right)$, where $T_{1} T_{2} \in \mathscr{T}^{\left(r_{1}, r_{3}\right)}$ is the product defined by joining two tangles as usual.

Like (1.4), let

$$
\widehat{Z}(T, \sigma, \tau)=\left(\gamma^{-m_{1}} \otimes \ldots \otimes \gamma^{-m_{k}}\right) \cdot\left(\lim _{\varepsilon \rightarrow 0} \varepsilon_{\sigma, r}{ }^{-1} Z\left(T_{\sigma, \tau, \varepsilon}\right) \varepsilon_{\tau, s}\right) .
$$

From the definition of $\widehat{Z}$ of pre-q-tangles, we get the following immediately from Theorem 1.5.

Theorem 2.5. For a pre-q-tangle $(T, \sigma, \tau), \widehat{Z}(T, \sigma, \tau)$ is an isotopy invariant of $(T, \sigma, \tau)$, i.e. $\widehat{Z}(T, \sigma, \tau)$ only depend on $\sigma, \tau$ and the isotopy type of $T$. Moreover, $\widehat{Z}$ gives a representation of the category of isotopy types of pre-q-tangles, in other words, $\widehat{Z}$ is compatible with the product of pre-q-tangles.

We call $\widehat{Z}(T, \sigma, \tau)$ a modified integral invariant of the pre-q-tangle $(T, \sigma, \tau)$.

To prove Theorem 2.2, we first show an analogy of Proposition 0.3 , which says that every pre-q-tangle $(T, \sigma, \tau)$ is a product of some simple pre-q-tangles. We give the integral of each simple pre-q-tangle exactly. We can compute the modified 
integral invariant $\widehat{Z}(T)$ from $\widehat{Z}$ of these simple pre-q-tangles as in Theorem 0.4 . We show that the category of q-tangles is a quotient category of pre-q-tangles in Sect. 4. We generalize $\widehat{Z}$ to a invariant of framed tangles $\widehat{Z}_{f}$ in Sect. 5 . From those, we get our main result Theorem 0.4 .

Proposition 2.6. Every pre-q-tangle is a product of tangles of the following types. The first type is a positive crossing $\left(R_{k}, \sigma, \sigma\right)$ with $\sigma(n-1)=k$. The second type is a negative crossing $\left(R_{k}^{-1}, \sigma, \sigma\right)$ with $\sigma(n-1)=k$. The next type is $\left(E_{k}, \sigma, \tau\right)$, where $\tau(n-1)=k, \sigma(i)=\tau(i)$ if $\tau(i)<k$ and $\sigma(i)=\tau(i)-2$ if $\tau(i)>k+1$, and its dual type $\left(E_{k}^{*}, \sigma, \tau\right), \tau(n-1)=k, \tau(i)=\sigma(i)$ if $\sigma(i)<k$ and $\tau(i)=\sigma(i)-2$ if $\sigma(i)>k+1$. The last type is $(I, \sigma, \tau)$ with $\sigma=(i i+$ $1) \tau$. In the above, $R_{k}, R_{k}^{-1}, E_{k}, E_{k}^{*}$ are tangles as in Fig. 2, and $I$ is a trivial tangle.

In the following, we prove Proposition 2.6 and Theorem 2.2. Kontsevich's integral for a tangle $T$ depends on the isotopy type and the positions of the end points of it. Therefore, the limit of the integral depends on the isotopy type of $T$ and the elements $\sigma, \tau$. We can decompose $T$ into a product of several simple tangles $R_{k}, R_{k}^{-1}, E_{k}$ and $E_{k}^{*}$ given in Fig. 2, which we call generator tangles. It is enough to show Theorem 2.2 for these generator tangles for all the possible $\sigma$ and $\tau$.

Let $T=R_{k} \in \mathscr{T}^{(r, s)}$ and $\alpha$ be a permutation such that $\alpha^{-1}(k)=|r|-1$. Then we have

$$
T_{\sigma, \tau, \varepsilon}=I_{\sigma, \alpha, \varepsilon} T_{\alpha, \alpha, \varepsilon} I_{\alpha, \tau, \varepsilon}
$$

Lemma 2.8 In the above situation, $\lim _{\varepsilon \rightarrow 0} \varepsilon_{\alpha} Z\left(T_{\alpha, \alpha, \varepsilon}\right) \varepsilon_{\alpha}{ }^{-1}=P_{k k+1} e^{r_{k} r_{k+1} \Omega_{k k+1} / 2}$, where $P_{k+1}$ denote the permutation diagram corresponding to $(k k+1)$ and $\Omega_{k+1}$ denote the chord connecting the two strings.

Proof. We first compute

$$
\lim _{\varepsilon_{|\gamma|-1} \rightarrow 0} \varepsilon_{|r|-1}{ }^{-r_{k} r_{k+1} \Omega_{k+1} /(2 \pi l)} Z\left(T_{\alpha, \alpha, \varepsilon}\right) \varepsilon_{|r|-1} s_{k} s_{k+1} \Omega_{k h+1} /(2 \pi l) .
$$

The strings of $T$ are parallel except the two strings forming the crossing. Hence, the integral for a configuration with a chord connecting these parallel strings is equal to 0 . The limit of the integral for a configuration with a chord connecting one of the parallel strings to one of the strings forming the crossing is equal to zero, because this integral has order $O\left(\varepsilon_{|r|-1}(\log \varepsilon)^{\ell}\right)$ for some $\ell$. The remaining case is a integral for configurations with chords connecting the two strings forming the crossing. We can assume that the levels of the bottom and top of $T$ are $t=0$ and $t=1$ respectively. Let us parametrize the two strings forming the crossing by $c+\varepsilon_{|r|-1} e^{l t}$ and $c-\varepsilon_{|r|-1} e^{l t}$, where $c$ is a real constant. The iterated integral for the configuration with $\ell$ chords connecting the two strings is $1 /\left(2^{\ell} \ell\right.$ !). Hence we get

$$
\lim _{\varepsilon_{|r|-1} \rightarrow 0} \varepsilon_{|r|-1}{ }^{-r_{k} r_{k+1} \Omega_{k+1} /(2 \pi l)} Z\left(T_{\alpha, \alpha, \varepsilon}\right) \varepsilon_{|r|-1} s^{s_{k+1} s_{k+1} \Omega_{k+1} /(2 \pi l)}=P_{k k+1} e^{r_{k} r_{k+1} \Omega_{k k+1} / 2} .
$$


Due to the 4-term relation, $\varepsilon_{\ell}{ }^{\Omega(\alpha, r, \ell)}$ commute with $\Omega_{k k+1}$ and $\varepsilon_{\ell}{ }^{\Omega(\alpha, r, \ell)} P_{k k+1}=$ $\varepsilon_{\ell}{ }^{\Omega(\alpha, s, \ell)} P_{k k+1}$ if $\ell<|r|-1$. Therefore,

$$
\begin{aligned}
\lim _{\varepsilon \rightarrow 0} \varepsilon_{\alpha, r}{ }^{-1} Z\left(T_{\alpha, \alpha, \varepsilon}\right) \varepsilon_{\alpha, s} & \\
= & \lim _{\varepsilon_{1} \rightarrow 0} \ldots \lim _{\varepsilon_{|r|-2} \rightarrow 0}\left(\prod_{\ell=1}^{|r|-2} \varepsilon_{\ell}{ }^{-\Omega(\alpha, r, \ell)}\right) P_{k k+1} e^{\Omega_{k k+1} / 2}\left(\prod_{\ell=1}^{|r|-2} \varepsilon_{\ell}^{\Omega(\alpha, r, \ell)}\right) \\
= & \lim _{\varepsilon_{1} \rightarrow 0} \ldots \lim _{\varepsilon_{|r|-2} \rightarrow 0} P_{k k+1} e^{\Omega_{k+1} / 2}=P_{k k+1} e^{\Omega_{k k+1} / 2} \cdot \square
\end{aligned}
$$

Similarly, for $T=R_{k}^{-1}$, we have

Lemma 2.11. With the notations in Lemma 2.8, we have $\lim _{\varepsilon \rightarrow 0} \varepsilon_{\alpha}{ }^{-1} Z\left(T_{\alpha, \alpha, \varepsilon}\right) \varepsilon_{\alpha}=$ $P_{k k+1} e^{-\Omega_{k k+1} / 2}$.

Let $T=E_{k}^{*} \in \mathscr{T}^{(r, s)}$ and $\alpha$ be a permutation such that $\alpha(|r|-1)=k$ and $\alpha(|r|-$ $2)=k+1$ if $k \leqq|r|-2$ or $k-1$ if $k \geqq|r|-1$ and $|r| \geqq 3$. Let $\beta$ be a permutation of degree $|r|-3$ such that $\beta^{-1}(\ell)=\alpha^{-1}(\ell)$ if $\ell \leqq<k$ and $\beta^{-1}(\ell)=$ $\alpha^{-1}(\ell+2)$ if $\ell \geqq k$. Then we have

$$
T_{\sigma, \tau, \varepsilon}=I_{\sigma, \alpha, \varepsilon} T_{\alpha, \beta, \varepsilon} I_{\beta, \tau, \varepsilon} .
$$

Lemma 2.13. In the above situation, $\lim _{\varepsilon \rightarrow 0} \varepsilon_{\alpha, r}{ }^{-1} Z\left(T_{\alpha, \beta, \varepsilon}\right) \varepsilon_{\beta, s}=E_{k}^{*} \in \mathscr{A}(T)$.

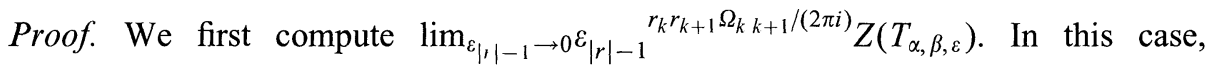
$r_{k} r_{k+1}=-1$ for any given orientations of the strings. We split $T$ to a product of $T_{1}$ and $T_{2}$, where $T_{1}$ is the tangle isotopic to $T$ and has distances $\varepsilon_{\beta^{-1}(\ell)}$ between the $\ell^{\text {th }}$

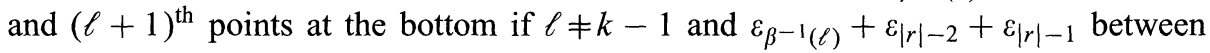
the $(k-1)^{\text {th }}$ and $k^{\text {th }}$ points. The strings of $T_{1}$ are parallel except the string with both end points at the top. Hence, the integral for a configuration with a chord connecting these parallel strings is equal to 0 . The limit of the integral for a configuration with a chord connecting one of the parallel strings to the string connecting two points at the top is equal to zero, because this integral has order $O\left(\varepsilon_{|r|-1}\right)$. The remaining case is a integral for configurations with chords connecting the string connecting two points at the top. However, this configuration is mapped to 0 due to the framing independence relation. Hence the only non-trivial integral is the integral for the configuration without any chords, and we get $\lim _{\varepsilon_{|r|-1} \rightarrow 0} Z\left(T_{1}\right)=E_{k}^{*}$. More precisely, $Z\left(T_{1}\right)=E_{k}^{*}+O\left(\varepsilon_{|r|-1}\right)$. From this fact, we get $\lim _{\varepsilon_{|r|-1} \rightarrow 0 \varepsilon_{|r|-1}} \Omega_{k k+1} /(2 \pi i) Z\left(T_{1}\right)=E_{k}^{*}$ since $E_{k}^{*} \Omega_{k+1}=0$ and the coefficient of $\varepsilon_{|r|-1} \Omega_{k+1} /(2 \pi l)$ for configurations with $p$ cords have order $O\left(\left(\log \varepsilon_{|r|-1}\right)^{P}\right)$. We also have

$$
\begin{aligned}
& \lim _{\varepsilon_{|r|-2} \rightarrow 0} \lim _{\varepsilon_{|,|-1} \rightarrow 0} \varepsilon_{|r|-2}{ }^{-\Omega(\alpha, r,|r|-2)} \varepsilon_{|r|-1} \Omega_{k k+1}^{/(2 \pi i)} Z\left(T_{1} T_{2}\right) \\
& =\lim _{\varepsilon_{|r|-2} \rightarrow 0} \varepsilon_{|r|-2}-\Omega(\alpha, r,|r|-2)\left(E_{k}^{*}+O\left(\varepsilon_{|r|-2}\right)\right) \\
& =\lim _{\varepsilon_{|r|-2} \rightarrow 0} \varepsilon_{|r|-2}{ }^{-\Omega(\alpha, r,|r|-2)} E_{k}^{*}=E_{k}^{*} .
\end{aligned}
$$


The last equality comes from the relation $\Omega_{k \ell} E_{k}^{*}=\Omega_{k+1 \ell} E_{i}^{*}$, since both configurations are the same cord diagrams. Now we have

$$
\lim _{\varepsilon_{1} \rightarrow 0} \ldots \lim _{\varepsilon_{|,|-3} \rightarrow 0} \varepsilon_{1}^{\Omega(\alpha, r, 1)} \ldots \varepsilon_{|r|-3}{ }^{\Omega(\alpha, r,|r|-3)} E_{k}^{*} \varepsilon_{|r|-3}{ }^{\Omega(\beta, s,|r|-3)} \ldots \varepsilon_{1}{ }^{\Omega(\beta, s, 1)}=E_{k}^{*},
$$

since $\varepsilon_{|r|-3}{ }^{\Omega(\alpha, r,|r|-3)} E_{k}^{*} \varepsilon_{|r|-3}{ }^{\Omega(\beta, s,|r|-3)}=E_{k}^{*}$.

Let $T \in \mathscr{T}^{(r, s)}$ be one of the generator tangles with a maximal point. Assume that the $k^{\text {th }}$ and $(k+1)^{\text {th }}$ points at the bottom of $T$ are connected by the string having the maximal point. Let $\alpha$ be a permutation such that $\alpha(|s|-1)=k$ and $\alpha(|s|-2)=k+1$ if $k \leqq|s|-2$ or $k-1$ if $k \geqq|r|-1$ and $|r| \geqq 3$. Let $\beta$ be a permutation of degree $|s|-3$ such that $\beta^{-1}(\ell)=, \alpha^{-1}(\ell)$ if $\ell \leqq<k$ and $\beta^{-1}(\ell)=$ $\alpha^{-1}(\ell+2)$ if $\ell \geqq k$. Then we have

$$
T_{\sigma, \tau, \varepsilon}=I_{\sigma, \beta, \varepsilon} T_{\beta, \alpha, \varepsilon} I_{\alpha, \tau, \varepsilon} .
$$

As in the previous case, we get the following lemma.

Lemma 2.15. In the above situation, $\lim _{\varepsilon \rightarrow 0} \varepsilon_{\beta, r}{ }^{-1} Z\left(T_{\alpha, \beta, \varepsilon}\right) \varepsilon_{\alpha, s}=E_{\imath} \in \mathscr{A}(T)$.

Now we discuss about the convergence of the modified integral for the trivial tangle $I_{\sigma, \tau, \varepsilon}$. Note that the integral $Z\left(I_{\sigma, \tau, \varepsilon}\right)$ may not be trivial. Since the symmetric group is generated by transpositions $(k k+1)$, we have

Lemma 2.16. $I_{\sigma, \tau, \varepsilon}$ is a product of tangles of the form $I_{\eta(k k+1), \eta, \varepsilon \text {, where } \eta \in S_{|r|-1}}$ and $1 \leqq k \leqq|r|-1$.

Proof of Theorem 0.3. First, note that usual tangle is expressed as a product of several tangles of forms as in Fig. 2. Hence (2.12), (2.14) and Lemma 2.16 imply Proposition 0.3.

Lemma 2.17. For a trivial tangle $I \in \mathscr{T}^{(r, r)}, \eta \in S_{|r|-1}$ and $1 \leqq k \leqq|r|-1$, $\lim _{\varepsilon \rightarrow 0} \varepsilon_{\eta(k k+1)}{ }^{-1} Z\left(I_{\eta(k k+1), \eta, \varepsilon}\right) \varepsilon_{\eta}$ is finite.

A proof for Lemma 2.17 is given in the next section.

Proof of Theorem 2.2. We showed that the modified integral of a tangle is a product of the modified integrals of the simple tangles of several types. We also proved the finiteness of the modified integral for these tangles. Hence we get the finiteness of the modified integral of any tangle.

\section{Modified Integral of Trivial Tangles}

The aim of this section is to prove Lemma 2.17, and give the actual form of $\lim _{\varepsilon \rightarrow 0} \varepsilon_{\eta(k k+1), r^{-1}} Z\left(I_{\eta(k k+1), \eta, \varepsilon}\right) \varepsilon_{\eta, r}$. We prepare several lemmas.

Lemma 3.1. Let $r=(1,1,1)$ and $I(a, b ; \varepsilon, a+b-\varepsilon) \in \mathscr{T}^{(r, r)}$ be a trivial tangle such that the distances of points at the top are $a$ and $b$, and those at the bottom are $\varepsilon$ and $a+b-\varepsilon$ as in Fig. 9. Then $\lim _{\varepsilon \rightarrow 0} Z(I(\varepsilon)) \varepsilon^{\Omega_{12} /(2 \pi i)}$ is finite, where $\Omega_{12}$ denote the cord connecting the first (left-most) and the second (middle) strings.

Proof. We place $I(a, b ; \varepsilon, a+b-\varepsilon)$ so that the level of the bottom is equal to $\varepsilon$ and that of the top is equal to $a$. Let $t \in \mathbf{R}$ be the parameter for the vertical 


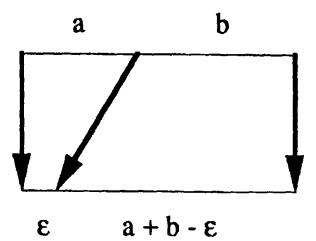

Fig. 9. Tangle $I(\varepsilon)$

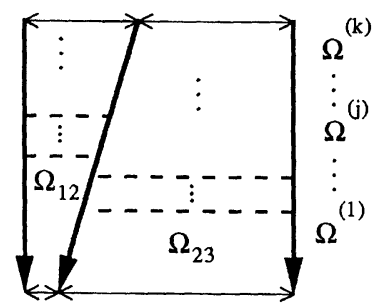

Fig. 10. Configuration $\Omega=\Omega^{(k)} \ldots \Omega^{(2)} \Omega^{(1)}$

coordinate and $y \in \mathbf{C}$ be the parameter for the horizontal coordinate. The first string is presented by $y=0$, the second string is presented by $y=t$ and the third string is presented by $y=a+b$. Let $\Omega_{i j}$ denote the chord connecting the $i^{\text {th }}$ and $j^{\text {th }}$ strings. The distance of the first and the third strings are constant and so the integral is equal to 0 .

We show the finiteness of the integral for any configurations with $\Omega_{12}$ and $\Omega_{23}$. Let $\Omega$ be a configuration of $\Omega_{12}$ and $\Omega_{23}$ as in Fig. 10. We denote this configuration by $\Omega=\Omega^{(r)} \ldots \Omega^{(2)} \Omega^{(1)}$, where $\Omega^{(1)}=\Omega_{23}$ and $\Omega^{(i)}=\Omega_{12}$ or $\Omega_{23}$ for $i \geqq 2$. Let $f_{t}(x)=1 / x$ if $\Omega^{(i)}=\Omega_{12}$ and $1 /(x-a-b)$ if $\Omega^{(i)}=\Omega_{23}$. Let $\mathbb{C}[[t]][\log t]$ be the set of polynomials in $\log t$ whose coefficients are series in $t$.

We compute the integral for the configuration $\Omega \Omega_{12}{ }^{p}$. Let $\int_{[a, b]}^{(k)} f_{*}\left(s_{*}\right) d s_{*}=$ $\int_{a}^{b} \int_{a}^{s_{k}} \ldots \int_{a}^{s_{3}} \int_{a}^{s_{2}} f_{k}\left(s_{k}\right) f_{k-1}\left(s_{k-1}\right) \ldots f_{1}\left(s_{1}\right) d s_{1} f_{2}\left(s_{2}\right) d s_{2} \ldots d s_{k-2} d s_{k-1} d s_{k}$ and $\int_{[a, b]}^{(k)}$ $f_{*}\left(s_{*}\right) d s_{*}=\int_{a}^{b} \int_{a}^{s_{k}} \ldots \int_{a}^{s_{3}} \int_{a}^{s_{2}} f_{k}\left(s_{k}\right) f_{k-1}\left(s_{k-1}\right) \ldots g\left(s_{1} f_{1}\left(s_{1}\right) d s_{1} f_{2}\left(s_{2}\right) d s_{2} \ldots d s_{k-2}\right.$ $d s_{k-1} d s_{k}$. Then the coefficient of $\Omega \Omega_{12}{ }^{k}$ in $Z(I(\varepsilon))$ is

$$
\sum_{k=0}^{\infty} \frac{1}{(2 \pi i)^{k+r}} \int_{[\varepsilon, a]}^{(r)} \int_{\left[\varepsilon, t_{1}\right]}^{(k)} \frac{d s_{*}}{s_{*}} f_{*}\left(t_{*}\right) d t_{*} \Omega \Omega_{12}{ }^{k} .
$$

On the other hand,

$$
\varepsilon^{\Omega_{12} /(2 \pi l)}=\sum_{k=0}^{\infty} \frac{1}{(2 \pi i)^{k} k !}(\log \varepsilon)^{k} \Omega_{12}{ }^{k}
$$

Hence the coefficient of $\Omega \Omega_{12}{ }^{p}$ in $Z(I(\varepsilon)) \varepsilon^{\Omega_{12} /(2 \pi i)}$ is given by

$$
\frac{1}{(2 \pi i)^{p+r}} \sum_{k+\ell=p[\varepsilon, a]} \int_{\left[\varepsilon, t_{1}\right]}^{(r)} \frac{d s_{*}}{s_{*}} f_{*}\left(t_{*}\right) d t_{*} \frac{1}{\ell !}(\log \varepsilon)^{\ell} \Omega \Omega_{12}^{p} .
$$


We have $\int_{\left[\varepsilon, t_{1}\right]}^{(k)} \frac{d s_{*}}{s_{*}}=\frac{1}{k !}\left(\log t_{1}-\log \varepsilon\right)^{k}$, and $\sum_{k+\ell=p} \frac{1}{k ! \ell !}(\log \varepsilon)^{\ell}\left(\log t_{1}-\log \varepsilon\right)^{k}$ $=\sum_{i+j+\ell=p} \frac{1}{i ! j ! \ell !}(\log \varepsilon)^{\ell}\left(\log t_{1}\right)^{l}(-1)^{j}(\log \varepsilon)^{j}=\sum_{l+q=p} \frac{1}{i ! q !}(\log \varepsilon-\log \varepsilon)^{q}$ $\left(\log t_{1}\right)^{l}=\frac{1}{p !}\left(\log t_{1}\right)^{p}$. Therefore,

$$
(3.4)=\frac{1}{(2 \pi i)^{p+r}} \int_{[\varepsilon, a]}^{(r)} \frac{1}{p !} f_{*}\left(t_{*}\right)\left(\log t_{1}\right)^{p} d t_{*} \Omega \Omega_{12}^{p} .
$$

The limit $\varepsilon \rightarrow 0$ of the above integral is finite because $f_{1}(x)=1 /(x-a-b)$ and $a+b>0$.

We get a similar lemma for the vertically and horizontally flipped tangles of $I(\varepsilon)$, and combining these results, we get the following.

Lemma 3.6. For the tangle $I(a-\varepsilon, \varepsilon ; \varepsilon, a-\varepsilon), \lim _{\varepsilon \rightarrow 0} \varepsilon^{-\Omega_{23} /(2 \pi i)} Z(I(a-\varepsilon, \varepsilon ; \varepsilon$, $a-\varepsilon)) \varepsilon^{\Omega_{12} /(2 \pi i)}$ is finite.

We can compute the integral for every configuration. Let $J=\left(p_{1}, q_{1}, \ldots, p_{g}, q_{g}\right)$ for positive integers $p_{1}, q_{1} \ldots, p_{g}, q_{g}, p(J)=\sum_{k=1}^{g} p_{k}, q(J)=\sum_{k=1}^{g} q_{k},|J|=p(J)$ $+q(J), g(J)=g, \Omega_{J}=\Omega_{12}{ }^{q_{4 j}} \Omega_{23}{ }^{p_{q j}} \ldots \Omega_{12}{ }^{q_{1}} \Omega_{23}{ }^{p_{1}}$, and

$$
\zeta_{J}=\zeta \underbrace{(1, \ldots, 1}_{p_{1}-1}, q_{1}+1, \underbrace{1, \ldots, 1}_{p_{2}-1}, q_{2}+1, \ldots, \underbrace{1, \ldots, 1}_{p_{q}-1}, q_{g}+1),
$$

where

$$
\zeta\left(s_{1}, \ldots, s_{k}\right)=\sum_{m_{1}<m_{2}<\cdots<m_{k} \in \mathrm{N}} \frac{1}{m_{1}^{s_{1}} m_{2}^{s_{2}} \ldots m_{k}^{s_{k}}} .
$$

$\zeta\left(s_{1}, \ldots, s_{k}\right)$ is called Zagier's multiple zeta values. Let $c_{J, k, \ell}$ be the numbers given by

$$
\begin{gathered}
c_{J, 0,0}=\frac{(-1)^{p(J)}}{(2 \pi i)^{|J|}} \zeta_{J}, \\
c_{J, 0, \ell}=(-1)^{\ell} \sum_{J^{\prime}} m_{J, J^{\prime}} c_{J^{\prime}, 0,0},
\end{gathered}
$$

where $J^{\prime}=\left(p_{1}^{\prime}, q_{1}^{\prime}, \ldots, p_{g^{\prime}}^{\prime}, q_{g^{\prime}}^{\prime}\right)$ and $\Omega_{J^{\prime}}$ runs over all substitution of $\ell$ copies of $\Omega_{12}$ to $\Omega_{J}$ except to the right of it, and $m_{J, J^{\prime}}$ is the number of ways to get $J^{\prime}$ from $J$ as above,

$$
c_{J, k, \ell}=(-1)^{k} \sum_{J^{\prime}} m_{J, J^{\prime}} c_{J^{\prime}, 0, \ell^{\prime}}
$$

where $\Omega_{J^{\prime}} \Omega_{12}{ }^{\ell^{\prime}}$ runs over all substitution of $k$ copies of $\Omega_{23}$ to $\Omega_{J} \Omega_{12}{ }^{\ell}$ except to the left of it, and $m_{J, J^{\prime}}$ is the number of ways to get $J^{\prime}$ from $J$ as above. For example, $c_{(1,1), 0,1}=-2 c_{(1,2), 0,0}=-\zeta(3)$, and $c_{(1,1), 1,1}=-2 c_{(2,1), 0,1}=2 c_{(1,1,1,1), 0,0}$ $+4 c_{(2,2), 0,0}=2 \zeta(2,2)+4 \zeta(1,3)$.

Proposition 3.8. For $I(1-\varepsilon, \varepsilon ; \varepsilon, 1-\varepsilon)$,

$$
\lim _{\varepsilon \rightarrow 0} \varepsilon^{-\frac{\Omega_{23}}{2 \pi l}} Z(I(1-\varepsilon, \varepsilon ; \varepsilon, 1-\varepsilon)) \varepsilon^{\frac{\Omega_{12}}{2 \pi l}}=\sum_{m=0}^{\infty} \sum_{g=0}^{[m / 2]} \sum_{\substack{h, S \geq 0 \\ y(J)=g \\ k+\ell+|J|=m}} c_{J, k, \ell} \Omega_{23}{ }^{k} \Omega_{J} \Omega_{12}{ }^{\ell},
$$

where we put $c_{J, 0,0}=1$ for empty sequence $J$. 
We denote the right-hand side of (3.9) by $\phi\left(\Omega_{12}, \Omega_{23}\right)$. Reformulating (3.9) by using (3.7), we get

$$
\begin{array}{r}
\phi\left(\Omega_{12}, \Omega_{23}\right)=\sum_{m=0}^{\infty} \sum_{g=0}^{[m / 2]} \sum_{\substack{g(J)=g \\
k+\ell+|J|=m}} c_{J, 0,0} \sum_{\begin{array}{c}
J^{\prime}=\left(p_{1}^{\prime}, \ldots, q_{g(J)}^{\prime}\right) \in Z_{\geqq 0}^{2 q(J)} \\
J^{\prime} \leqq J(\text { component wise) }
\end{array}}(-1)^{\left|J^{\prime}\right|} \times \\
\prod_{j=1}^{g(J)}\left(\begin{array}{c}
p_{j} \\
p_{j}^{\prime}
\end{array}\right)\left(\begin{array}{c}
q_{j} \\
q_{j}^{\prime}
\end{array}\right) \Omega_{23}{ }^{k} \Omega_{J-J^{\prime}} \Omega_{12}{ }^{\ell} .
\end{array}
$$

Proof of Theorem 3.8. Let $\Omega_{J}=\Omega^{(|J|)} \ldots \Omega^{(2)} \Omega^{(1)}$, where $\Omega^{(k)}=\Omega_{12}$ or $\Omega_{23}$, and let $f_{i}(x)=1 / x$ if $\Omega^{(i)}=\Omega_{12}$ and $1 /(x-1)$ if $\Omega^{(i)}=\Omega_{23}$. First we prove for the case $k=\ell=0$. The coefficient for the configuration $\Omega_{J}$ in the integral $\lim _{\varepsilon \rightarrow 0}$ $\varepsilon^{-\Omega_{23} /(2 \pi i)} Z(I(1-\varepsilon, \varepsilon ; \varepsilon, 1-\varepsilon)) \varepsilon^{\Omega_{12} /(2 \pi i)}$ is equal to that in $\lim _{\varepsilon \rightarrow 0} Z(I(1-\varepsilon, \varepsilon ; \varepsilon$, $1-\varepsilon)$ ), and it is equal to the iterated integral

$$
\int_{[0,1]}^{(|J|)} f_{*}\left(s_{*}\right) d s_{*}
$$

Note that $f_{1}(x)=1 /(x-1)$ and

$$
\begin{aligned}
& \int_{0}^{x} \sum_{m_{1}<\cdots<m_{k} \in \mathrm{N}} \frac{t^{m_{k}}}{m_{1}^{s_{1}} \ldots m_{k}^{s_{k}}} \frac{d t}{t}=\sum_{m_{1}<\cdots<m_{k} \in \mathrm{N}} \frac{x^{m_{k}}}{m_{1}^{s_{1}} \ldots m_{k}^{s_{k}+1}}, \\
& \int_{0}^{x} \sum_{m_{1}<\cdots<m_{k} \in \mathrm{N}} \frac{t^{m_{k}}}{m_{1}^{s_{1}} \ldots m_{k}^{s_{k}}} \frac{d t}{t-1}=\sum_{m_{1}<\cdots<m_{k}<m_{k+1} \in \mathrm{N}} \frac{x^{m_{k+1}}}{m_{1}^{s_{1}} \ldots m_{k}^{s_{k}} m_{k+1}} .
\end{aligned}
$$

By using these relations inductively to compute (3.11), we get the right-hand side of (3.7a).

We reduce the other cases to the $k=\ell=0$ case. Since

$$
\begin{aligned}
\varepsilon^{\Omega_{12} /(2 \pi i)} & =\sum_{k=0}^{\infty} \frac{1}{(2 \pi i)^{k}} \int_{[1, \varepsilon]} \frac{d s_{*}}{s_{*}}, \\
\varepsilon^{-\Omega_{12} /(2 \pi i)} & =\sum_{k=0}^{\infty} \frac{1}{(2 \pi i)^{k}} \int_{[1-\varepsilon, 0]} \frac{d s_{*}}{s_{*}-1},
\end{aligned}
$$

the coefficient of $\Omega_{J, k, \ell}$ of the integral $\varepsilon^{-\Omega_{23} /(2 \pi l)} Z(I(1-\varepsilon, \varepsilon ; \varepsilon, 1-\varepsilon)) \varepsilon^{\Omega_{12} /(2 \pi l)}$ is given by

$$
\begin{gathered}
\frac{1}{(2 \pi i)^{k+\ell+|J|}} \sum_{n=0 m=0[1-\varepsilon, \varepsilon]}^{k} \sum_{\frac{1}{\ell} \int^{(k-n)} \frac{d x_{*}}{x_{*}-1} \int_{[\varepsilon, 1-\varepsilon]}^{(\ell-m)} \frac{d y_{*}}{y_{*}} \int_{\left[\varepsilon, u_{1}\right]}^{(|J|)} f_{*}\left(s_{*}\right) d s_{*} \times} \\
\int_{[1-\varepsilon, \varepsilon]}^{(n)} \frac{d u_{*}}{u_{*}-1} \int_{[1, \varepsilon]}^{(m)} \frac{d v_{*}}{v_{*}} .
\end{gathered}
$$

The iterated integral satisfies the following:

$$
\int_{[a, b]}^{(k)} \int_{\left[a, t_{1}\right]}^{(\ell)} f_{*}\left(s_{*}\right) d s_{*} g_{*}\left(t_{*}\right) d t_{*}=\int_{[a, b]}^{(\ell)} \int_{\left[s_{1}, b\right]}^{(k)} g_{*}\left(t_{*}\right) d t_{*} f_{*}\left(s_{*}\right) d s_{*} .
$$


This implies

$$
\begin{aligned}
(3.13)= & \frac{1}{(2 \pi i)^{k+\ell+|J|}} \sum_{0 \leqq n \leqq k[\varepsilon, 1-\varepsilon]} \int_{\left[\varepsilon, u_{1}\right]}^{(n)} \int_{0 \leqq m \leqq \ell}^{(|J|)}\left(\int_{\left[\varepsilon, s_{1}\right]}^{(\ell-m)} \frac{d y_{*}}{y_{*}} \int_{[1, \varepsilon]}^{(m)} \frac{d v_{*}}{v_{*}}\right) \times \\
& f_{*}\left(s_{*}\right) d s_{*} \frac{d u_{*}}{u_{*}-1} \int_{[1-\varepsilon, 0]}^{(k-n)} \frac{d x_{*}}{x_{*}-1} \\
= & \frac{(-1)^{k+\ell}}{(2 \pi i)^{k+\ell+|J|}} \int_{[\varepsilon, 1-\varepsilon]}^{(|J|)} \int_{\left[0, s_{1}\right]}^{(k)} \frac{d u_{*}}{u_{*}-1} \int_{\left[s_{1}, 1\right]}^{(\ell)} \frac{d v_{*}}{v_{*}} f_{*}\left(s_{*}\right) d s_{*} .
\end{aligned}
$$

Hence the limit of $(3.13)$ is

$$
\frac{(-1)^{k+\ell}}{(2 \pi i)^{k+\ell+|J|}} \int_{\left[0, u_{1}\right]}^{(|J|)} \int_{\left[0, s_{1}\right]}^{(k)} \frac{d u_{*}}{u_{*}-1} \int_{\left[s_{1}, 1\right]}^{(\ell)} \frac{d v_{*}}{v_{*}} f_{*}\left(s_{*}\right) d s_{*} .
$$

First split the integral intervals of $u_{1}, \ldots, u_{k}$ by $s_{1}, \ldots, s_{|J|}$, and then split the integral intervals of $v_{1}, \ldots, v_{\ell}$ by $s_{1}, \ldots, s_{|J|}, u_{1}, \ldots, u_{k}$. Then the above integral is a sum of iterated integrals and each integral is expressed by $c_{J, 0,0}$ for some $J$. Considering which $J$ corresponds to a part of the integral, we get the proposition.

Now we introduce an operator $\Delta$ which duplicates a string of a tangle. Let $T \in \mathscr{T}^{(r, s)}$ be a tangle without maximal nor minimal points. Let $w$ be a string of $T$ connecting the $k^{\text {th }}$ point at the top and the $j^{\text {th }}$ point at the bottom. Let $T^{\prime}$ be the tangle obtained by adding an extra string $w^{\prime}$ to $T$ which is close and parallel to $w$. The orientation of $w^{\prime}$ is equal to that of $w$. We denote this operator by $\Delta_{k}$. Then $\Delta_{k}\left(T_{\sigma, \tau, \varepsilon}\right)=T_{\sigma^{\prime}, \tau^{\prime}, \varepsilon}^{\prime}$, where $\sigma^{\prime}(\ell)=\sigma(k)$ if $\sigma(\ell) \leqq k, \sigma^{\prime}(|s|)=k, \sigma^{\prime}(\ell)=$ $\sigma(\ell)+1$ if otherwise, and $\tau^{\prime}(\ell)=\tau(\ell)$ if $\sigma(\ell) \leqq j, \tau^{\prime}(|r|)=j, \tau^{\prime}(\ell)=\tau(\ell)+1$ if otherwise. We can compute the modified integral of $\Delta_{k}\left(T_{\sigma, \tau, \varepsilon}\right)$ from the modified integral of $T_{\sigma, \tau, \varepsilon}$ as follows.

Proposition 3.15. We have

$$
\lim _{\varepsilon \rightarrow 0} \varepsilon_{\sigma^{\prime}, r^{\prime}}^{-1} \Delta_{k}\left(T_{\sigma^{\prime}, \tau^{\prime}, \varepsilon}^{\prime}\right) \varepsilon_{\tau^{\prime}, s^{\prime}}=\lim _{\varepsilon \rightarrow 0} \varepsilon_{\sigma, r}{ }^{-1} Z\left(T_{\sigma, \tau, r}\right) \varepsilon_{\tau, s} \mid \Omega_{w r}=\Omega_{w r}+\Omega_{w w^{\prime} t}
$$

for every string $v$ of $T$ other than $w$.

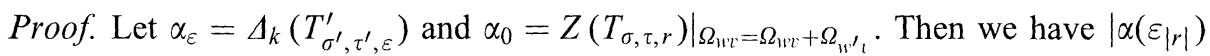
$-\alpha(0) \mid<O\left(\varepsilon_{|r|}\right)$. Since the coefficient of $\varepsilon_{|r|} \Omega_{1 w w^{\prime}} /(2 \pi t)$ for the configuration with $\ell$ cords has order $\left(\log \varepsilon_{|r|}\right)^{\ell}$, we have

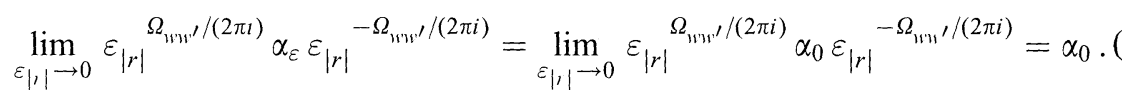

For the last equality, we use the 4-term relation $\left[\Omega_{w w^{\prime}}, \Omega_{w v}+\Omega_{w^{\prime} v}\right]=0$. The formula (3.16) comes from (3.17).

Lemma 3.18. For $I(a-\varepsilon, \varepsilon ; \varepsilon, a-\varepsilon)$,

$$
\begin{gathered}
\lim _{a \rightarrow 0} a^{-\frac{\Omega_{12}+\Omega_{13}}{2 \pi l}}\left(\lim _{\varepsilon \rightarrow 0} \varepsilon^{-\frac{\Omega_{23}}{2 \pi l}} Z(I(a-\varepsilon, \varepsilon ; \varepsilon, a-\varepsilon)) \varepsilon^{\frac{\Omega_{12}}{2 \pi l}}\right) a^{\frac{\Omega_{13}+\Omega_{23}}{2 \pi l}} \\
=\lim _{\varepsilon \rightarrow 0} \varepsilon^{-\frac{\Omega_{23}}{2 \pi i}} Z(I(1-\varepsilon, \varepsilon ; \varepsilon, 1-\varepsilon)) \varepsilon^{\frac{\Omega_{12}}{2 \pi l}} .
\end{gathered}
$$




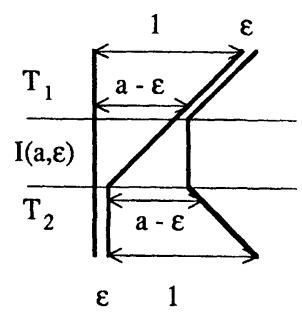

Fig. 11. Product of tangles $I(\varepsilon, a), T$, and $T$

Proof. Consider a product $T_{1} I(a-\varepsilon, \varepsilon ; \varepsilon, a-\varepsilon) T_{2}$ of three tangles in Fig. 11. Let $\mathscr{A}_{0}^{(k)}(X)$ denote the subspace of $\mathscr{A}_{0}(X)$ spanned by cord diagrams with $k$ cords and $p_{k}: \mathscr{A}_{0}(X) \rightarrow \mathscr{A}_{0}^{(k)}(X)$ be the natural projection. Form (3.12) and proof of Proposition 3.15, we have

$$
\begin{gathered}
p_{k}\left(a^{-\left(\Omega_{12}+\Omega_{13}\right) /(2 \pi i)}\right)=p_{k}\left(\varepsilon^{-\Omega_{23} /(2 \pi i)} Z\left(T_{1}\right) \varepsilon^{\Omega_{23} /(2 \pi i)}\right)+O\left(\varepsilon(\log \varepsilon)^{k}\right), \\
p_{k}\left(a^{\left(\Omega_{13}+\Omega_{23}\right) /(2 \pi i)}\right)=p_{k}\left(\varepsilon^{-\Omega_{12} /(2 \pi i)} Z\left(T_{1}\right) \varepsilon^{\Omega_{12} /(2 \pi i)}\right)+O\left(\varepsilon(\log \varepsilon)^{k}\right) .
\end{gathered}
$$

Hence

$$
\begin{aligned}
& p_{k}\left(a^{-\frac{\Omega_{12}+\Omega_{13}}{2 \pi i}}\left(\lim _{\varepsilon \rightarrow 0} \varepsilon^{-\frac{\Omega_{23}}{2 \pi i}} Z(I(a-\varepsilon, \varepsilon ; \varepsilon, a-\varepsilon)) \varepsilon^{\frac{\Omega_{12}}{2 \pi l}}\right) a^{\frac{\Omega_{13}+\Omega_{23}}{2 \pi l}}\right) \\
& \quad=\lim _{\varepsilon \rightarrow 0} p_{k}\left(a^{-\frac{\Omega_{12}+\Omega_{13}}{2 \pi i}} \varepsilon^{-] f r a c \Omega_{23} 2 \pi i} Z(I(a-\varepsilon, \varepsilon ; \varepsilon, a-\varepsilon)) \varepsilon^{\frac{\Omega_{12}}{2 \pi i}} a^{\frac{\Omega_{13}+\Omega_{23}}{2 \pi i}}\right) \\
& \quad=\lim _{\varepsilon \rightarrow 0}\left(p_{k}\left(\varepsilon^{\frac{-\Omega_{23}}{2 \pi l}} Z\left(T_{1}\right) Z(I(a-\varepsilon, \varepsilon ; \varepsilon, a-\varepsilon)) Z\left(T_{2}\right) \varepsilon^{\frac{\Omega_{12}}{2 \pi l}}\right) O\left(\varepsilon(\log \varepsilon)^{k}\right)\right) \\
& \quad=\lim _{\varepsilon \rightarrow 0} p_{k}\left(\varepsilon^{-\frac{\Omega_{23}}{2 \pi i}} Z(I(1-\varepsilon, \varepsilon ; \varepsilon, 1-\varepsilon)) \varepsilon^{\frac{\Omega_{12}}{2 \pi l}}\right),
\end{aligned}
$$

since the tangle $T_{1} I(a-\varepsilon, \varepsilon ; \varepsilon, a-\varepsilon) T_{2}$ is isotopic to $I(1-\varepsilon, \varepsilon ; \varepsilon, 1-\varepsilon)$ with the same endpoints. The last form does not depend on $a$ and so we get (3.19).

Let

$$
\phi\left(\Omega_{12}, \Omega_{23}\right)=\sum_{m=0}^{\infty} \sum_{g=0}^{[m / 2]} \sum_{\begin{array}{c}
k, \ell \geq 0 \\
g(J)=g \\
k+\ell+|J|=m
\end{array}} c_{J, k, \ell} \Omega_{23}{ }^{k} \Omega_{J} \Omega_{12}{ }^{\ell} .
$$

Then, by (3.9),

$$
\phi\left(\Omega_{12}, \Omega_{23}\right)=\lim _{\varepsilon \rightarrow 0} \varepsilon^{-\Omega_{23} /(2 \pi i)} Z(I(1-\varepsilon, \varepsilon ; \varepsilon, 1-\varepsilon)) \varepsilon^{\Omega_{12} /(2 \pi i)}
$$

Theorem 3.22. Let $I$ be the trivial tangle in $\mathscr{T}^{(r, r)}$ for some $r$ and let $\eta$ be a permutation of $|r|-1$ letters. Then the limit

$$
\lim _{\varepsilon \rightarrow 0} \varepsilon_{\eta(k k+1), r}{ }^{-1} Z\left(I_{\eta(k k+1), \eta, \varepsilon}\right) \varepsilon_{\eta, r}=I \in \mathscr{A}(I)
$$

if there is $j<k$ between $\eta(k)$ and $\eta(k+1)$, 


$$
\begin{aligned}
& \lim _{\varepsilon \rightarrow 0} \varepsilon_{\eta(k k+1), r^{-1}} Z\left(I_{\eta(k k+1), \eta, \varepsilon}\right) \varepsilon_{\eta, r}
\end{aligned}
$$

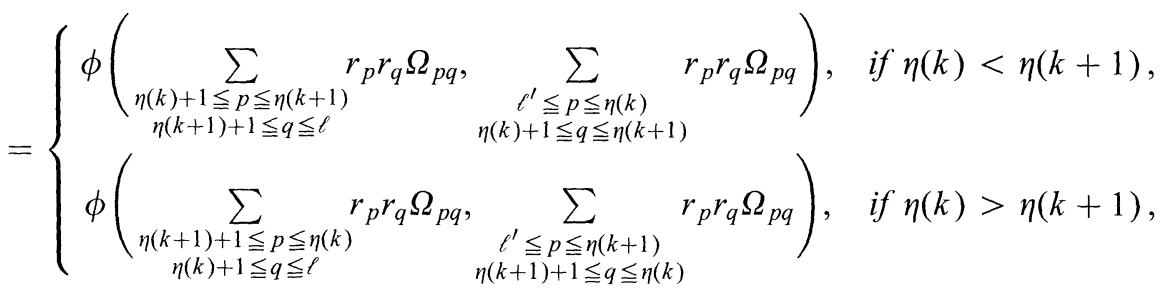

where $\ell$ is the maximal number such that $\eta^{-1}(q) \geqq \ell^{\prime}+1$ for all $q$ such that $\max (\eta(k), \eta(k+1))+1 \leqq q \leqq \ell$, and $\ell^{\prime}$ is the minimal number such that $\eta^{-1}(p)$ $\geqq k$ for all $\ell^{\prime} \leqq p \leqq \min (\eta(k), \eta(k+1))$.

Proof. We prove the above for $r=(1,1, \ldots, 1)$. Results for other cases come from this case by changing the signatures corresponding to the factor $(-1)^{P} \uparrow$ in Definition 1.1. Assume that $\varepsilon_{k}$ is very small for $k>i+1$. Then the strings of $I_{\eta(k k+1), \eta, \varepsilon}$ are split into $k+2$ groups, in which strings are parallel and very close. Let $G_{1}, G_{2}, \ldots, G_{k+2}$ be those groups from left to right. Let $r^{\prime}$ be a sequence of $k+1$ ones and $I^{\prime}$ be the trivial tangle in $\mathscr{T}^{\left(r^{\prime}, r^{\prime}\right)}$. Then, there is $\eta^{\prime} \in S_{k+1}$ such that $I_{\eta^{\prime}(k k+1), \eta^{\prime}, \varepsilon}^{\prime}$ is the modified tangle obtained by replacing each group $G_{\ell}$ by a single string. Let $\Omega^{(j \ell)}=\sum_{s \in G_{j}, t \in G_{l}} \Omega_{s t}$ where $\Omega_{s t}$ denote the cord connecting the strings $s$ and $t$. Proposition 3.18 implies that we can get $\lim _{\varepsilon \rightarrow 0} \varepsilon_{\eta(k k+1), r}{ }^{-1} Z\left(I_{\eta(k k+1), \eta, \varepsilon}\right)$ $\varepsilon_{\eta, r}$ from $\lim _{\varepsilon \rightarrow 0} \varepsilon_{\eta^{\prime}(k k+1), r^{\prime}}{ }^{-1} Z\left(I_{\eta^{\prime}(k k+1), \eta^{\prime}, \varepsilon}^{\prime}\right) \varepsilon_{\eta^{\prime}, r^{\prime}}$ by changing each string to strings in the corresponding group and each cord $\Omega_{j \ell}$ connecting the $j^{\text {th }}$ and $\ell^{\text {th }}$ strings of $I^{\prime}$ to a sum of cords $\Omega^{(\ell \ell)}$.

From now on, we investigate $\lim _{\varepsilon \rightarrow 0} \varepsilon_{\eta^{\prime}(k k+1), r^{\prime}}-1 Z\left(I_{\eta^{\prime}(k k+1), \eta^{\prime}, \varepsilon}^{\prime}\right) \varepsilon_{\eta^{\prime}, r^{\prime}}$. Let $j$ be an integer with $0<j<\eta(k)$ or $\eta(k+2)<j \leqq k+2$. Then the coefficient of a configuration containing $\Omega_{j \eta(k)}, \Omega_{j \eta(k)+1}, \ldots$, or $\Omega_{j \eta(k+1)+1}$ is 0 in the limit of the modified integral since such a coefficient in $\varepsilon_{\eta^{\prime}(k k+1), r^{\prime}}{ }^{-1} Z\left(I_{\eta^{\prime}(k k+1), \eta^{\prime}, \varepsilon}^{\prime}\right) \varepsilon_{\eta^{\prime}, r^{\prime}}$ is 0 or bounded by $O\left(\varepsilon_{k}\left(\log \varepsilon_{k}\right)^{\ell}\right)$ for some $\ell$.

Now consider the case $\eta(k)+1<\eta(k+1)$. Let $\eta(k)+1<j \leqq \eta(k+1)+1$. Then the coefficient of a configuration containing $\Omega_{j \eta(k)}$ or $\Omega_{j \eta(k)+1}$ is 0 in the limit of the modified integral as before. Let $\eta(k)+1<\ell \leqq \eta(k+1)+1$. Then the coefficient of a configuration containing $\Omega_{j \eta(k)}$ or $\Omega_{j \eta(k)+1}$ is also 0 in the limit of the modified integral as before. Hence, the limit has a nontrivial coefficient for configurations consisting of $\Omega_{\eta(k) \eta(k)+1}$ and $\Omega_{\eta(k+1) \eta(k+1)+1}$. We can compute the coefficient of $\Omega_{\eta(k) \eta(k)+1}{ }^{p} \Omega_{\eta(k+1) \eta(k+1)+1}{ }^{q}$ in $Z\left(I_{\eta^{\prime}(k k+1), \eta^{\prime}, \varepsilon}\right)$, and it is $\frac{1}{(2 \pi l)^{p+q} p ! q !}$ $\left(\log \varepsilon_{k}-\log \varepsilon_{k+1}\right)^{p}\left(\log \varepsilon_{k+1}-\log \varepsilon_{k}\right)^{q}$. Therefore, the coefficient of $\Omega_{\eta(k) \eta(k)+1}{ }^{p}$ $\Omega_{\eta(k+1) \eta(k+1)+1}{ }^{q}$ in the modified integral $\varepsilon_{\eta^{\prime}(k k+1), r^{\prime}}{ }^{-1} Z\left(I_{\eta^{\prime}(k k+1), \eta^{\prime}, \varepsilon}^{\prime}\right) \varepsilon_{\eta^{\prime}, r^{\prime}}$ is

$$
\begin{aligned}
\frac{1}{(2 \pi i)^{p+q}} \sum_{\substack{r_{1}+r_{2}+r_{3}=p \\
s_{1}+s_{2}+s_{3}=q}} \frac{1}{s_{1} ! s_{2} ! s_{3} ! r_{1} ! r_{2} ! r_{3} !}\left(-\log \varepsilon_{k}\right)^{r_{1}}\left(-\log \varepsilon_{k+1}\right)^{s_{1}} \times \\
\left(\log \varepsilon_{k}-\log \varepsilon_{k+1}\right)^{r_{2}}\left(\log \varepsilon_{k+1}-\log \varepsilon_{k}\right)^{s_{2}}\left(\log \varepsilon_{k+1}\right)^{r_{3}}\left(\log \varepsilon_{k}\right)^{s_{3}} \\
= \begin{cases}1 & \text { if } p=q=0, \\
0 & \text { if otherwise. }\end{cases}
\end{aligned}
$$


This implies that $\lim _{\varepsilon \rightarrow 0} \varepsilon_{\eta^{\prime}(k k+1), r^{\prime}}^{-1} Z\left(I_{\eta^{\prime}(k k+1), \eta^{\prime}, \varepsilon}^{\prime}\right) \varepsilon_{\eta^{\prime}, r^{\prime}}=I^{\prime} \in \mathscr{A}\left(I^{\prime}\right)$, and so

$$
\lim _{\varepsilon \rightarrow 0} \varepsilon_{\eta(k k+1), r^{-1}} Z\left(I_{\eta(k k+1), \eta, \varepsilon}\right) \varepsilon_{\eta, r}=I \in \mathscr{A}(I) .
$$

If $\eta(k+1)+1<\eta(k)$, we get the same result.

If $\eta(k)+1=\eta(k+1)$ or $\eta(k+1)+1=\eta(k)$, we get

$$
\lim _{\varepsilon \rightarrow 0} \varepsilon_{\eta^{\prime}(k k+1), r^{\prime}}-1 \quad Z\left(I_{\eta^{\prime}(k k+1), \eta^{\prime}, \varepsilon}^{\prime}\right) \varepsilon_{\eta^{\prime}, r^{\prime}}=\phi\left(\Omega_{\eta^{\prime}(k+1) \eta^{\prime}(k+1)+1}, \Omega_{\eta^{\prime}(k) \eta^{\prime}(k)+1}\right)
$$

from (3.16) and (3.21). Hence, by substituting $\Omega_{j l}=\Omega^{(j l)}$, we get (3.23b).

This theorem implies the lemma at the end of the last section.

Remark. 3.26. Lemma 2.8, Lemma 2.11 and (3.23) give modified integrals for simple tangles in Proposition 2.6.

\section{Modified Integral for $q-$ Tangles}

In Sect. 2 and Sect. 3, we investigate the modified integral for pre-q-tangles. Here, we show that the category of q-tangles is a quotient of the category of pre-qtangles. We also show that the results for pre-q-tangles in the last two sections can be translated for q-tangles.

First, we give a mapping $f$ from pre-q-tangles to q-tangles. Let $r$ and $s$ be sequences of $\pm 1, T \in \mathscr{T}^{(r, s)}$ and $\sigma \in S_{|s|}, \tau \in S_{|r|}$. We define

$$
f((T, \sigma, \tau))=\left(T, f_{w}(\sigma), f_{w}(\tau)\right) \quad(\mathrm{q} \text {-tangle }),
$$

where $f_{w}(\sigma)$ and $f_{w}(\tau)$ are non-associative words with supports $r$ and $s$ defined by the following. For the sequence $r=\left(r_{1}, r_{2}, \ldots, r_{|r|}\right)$, first put a bracket to group $r_{\sigma(|r|-1)} r_{\sigma(|r|-1)+1}$. At the $k^{\text {th }}$ step, put a bracket to a subsequence $\left(w_{1} w_{2}\right)$, where $w_{1}$ is the group containing $r_{\sigma(|r|-k)}$ as the right-most element and $w_{2}$ is the group next to $w_{1}$. Repeating this procedure to $(|r|-1)^{\text {th }}$ step, we get a non-associative word and we denote it by $f_{w}(\sigma)$. We similarly define $f_{w}(\tau)$. Since the families of morphisms of the categories of pre-q-tangles and q-tangles are identical, the definition of the mapping $f$ immediately implies the following.

Proposition 4.2. The mapping $f$ induces a surjective functor from the category of pre-q-tangles to the category of q-tangles.

In the rest of this section, we show the following.

Proposition 4.3. The mapping $f$ induces a representation of $q$-tangles from the modified integral for pre-q-tangles in the last two sections.

Proof. We show that the modified integrals of pre-q-tangles $\left(T, \sigma_{1}, \tau_{1}\right)$ and $\left(T, \sigma_{2}, \tau_{2}\right)$ are equal if $f_{w}\left(\sigma_{1}\right)=f_{w}\left(\sigma_{2}\right)$ and $f_{w}\left(\tau_{1}\right)=f_{w}\left(\tau_{2}\right)$. Since $\left(T, \sigma_{2}, \tau_{2}\right)=$ $\left(I, \sigma_{2}, \sigma_{1}\right)\left(T, \sigma_{1}, \tau_{1}\right)\left(I, \tau_{1}, \tau_{2}\right)$, it is enough to show that the modified integral for $(I, \sigma, \tau)$ is equal to $I$ if $f_{w}(\sigma)=f_{w}(\tau)$. Then the following lemma and (3.24) implies the proposition.

Lemma 4.4. Let $\sigma$ and $\tau$ be two permutations of $n-1$ letters. Then $f_{w}(\sigma)=f_{w}(\tau)$ if and only if there are sequences of permutations $\sigma=\eta_{0}, \ldots, \eta_{l}=\tau$ and integers 
$k_{1}, \ldots, k_{\ell}$ satisfying $\left(^{*}\right) \eta_{l+1}=\eta_{i}\left(k_{l}, k_{i}+1\right)$ and there is some $j_{i}<k_{l}$ between $\eta_{l}\left(k_{i}\right)$ and $\eta_{i}\left(k_{i}+1\right)$.

This lemma comes from a combinatorial argument. We omit the proof.

Proof of Theorem 0.4. By Remark 3.27, $\hat{Z}_{f}$ in Theorem 0.4 for simple tangles in Proposition 0.3 coincide with the representations in Proposition 4.3 for those tangles.

\section{Invariant for Framed Tangles}

In this section, we construct an invariant of oriented tangles with blackboard framing (see for example [13]). Let $T$ be a tangle. In this section we use chord diagrams $\mathscr{A}(T)$ instead of $\mathscr{A}_{0}(T)$. It means that we do not impose the framing independence relation given in Fig. 1 (b). We construct an invariant of framed q-tangles with values in $\mathscr{A}(T)$. Kontsevich's integral for a tangle becomes infinity at neighborhoods of maximal and minimal points if we don't have the framing independence relation. We normalize the integral at maximal and minimal points so that it is finite.

Let $T$ be a tangle with only one minimal or maxima point $p$. For small positive $\varepsilon \in \mathbf{R}$, let $T_{\varepsilon}$ be the tangle obtained from $T$ by cutting a part near $p$ by a horizontal plane. Here $\varepsilon$ is the distance between two intersection points of the distinguished string containing $p$ with the cutting horizontal plane. Then $T=T_{\varepsilon} \times\left(T-T_{\varepsilon}\right)$ and $T_{\varepsilon}$ is a trivial tangle. We can define $Z\left(T_{\varepsilon}\right) \in \mathscr{A}\left(T_{\varepsilon}\right)$. Let $\omega^{d}$ stand for a chord diagram in $\mathscr{A}\left(T-T_{\varepsilon}\right)$ which consists of $d$ parallel dashed lines near $p$ and connecting points of the distinguished string as in Fig. 12. We regard $\omega^{d}$ as the formal $d^{\text {th }}$ power of $\omega$.

Proposition 5.1. Let $T$ be a tangle with just one extremal point $p$. If $p$ is a minimal point, there exist

$$
Z_{f}(T)=\lim _{\varepsilon \rightarrow 0} Z\left(T_{\varepsilon}\right) \varepsilon^{-\omega /(2 \pi i)} \in \mathscr{A}(T)
$$

If $p$ is a minimal point, there exist

$$
Z_{f}(T)=\lim _{\varepsilon \rightarrow 0} \varepsilon^{\omega /(2 \pi l)} Z\left(T_{\varepsilon}\right) \in \mathscr{A}(T)
$$

Proof. We use a similar argument in the proof of Lemma 3.1. We prove for the minimal point case. We may assume that the bottom plane of $T$ contains the minimal point $p$. Let $T^{\prime}$ be a tangle obtained from $T$ by a horizontal move, keeping each point of the top and the bottom planes invariant. Due to the 4-term relation, $Z_{f}\left(T^{\prime}\right)=Z_{f}(T)$ if $Z_{f}(T)$ is finite. Hence it is enough to show the finiteness for the tangle $T$ in Fig. 13.

We compute the coefficient of the integral $Z\left(T_{\varepsilon}\right) \varepsilon^{-\omega /(2 \pi i)}$ for the configuration $\Omega^{(r)} \ldots \Omega^{(2)} \Omega^{(1)} \omega^{p}$. Recall that we assumed every tangle is contained in $\mathbf{R} \times \mathbf{R} \subset \mathbf{R} \times \mathbf{C}$ except neighborhoods of crossing points. Hence, $T_{\varepsilon} \subset \mathbf{R} \times \mathbf{R}$. As in the proof of Lemma 3.1, this notation stands for a configuration with $\Omega^{(r)}, \ldots, \Omega^{(1)}$ from top to bottom in turns and the lowermost part is $\omega^{p}$. Let $w_{k}^{(1)}$ and $w_{k}^{(2)}$ 


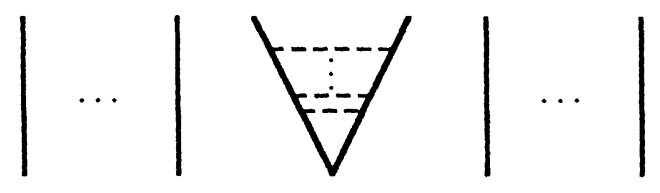

Fig. 12. Cord diagram $\omega^{d}$

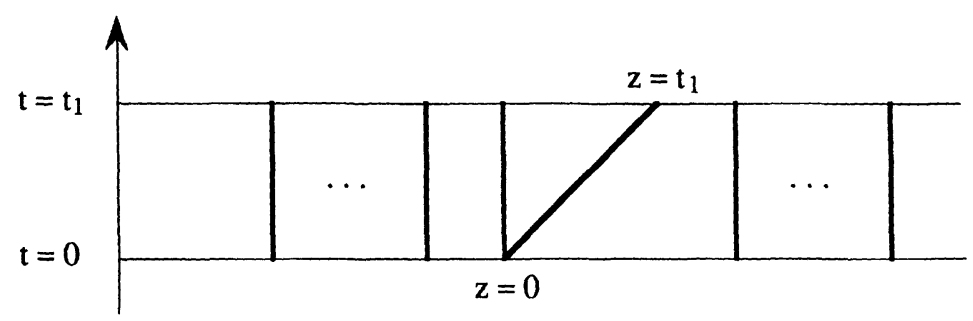

Fig. 13.

be the two string of $T$ containing the end points of $\Omega^{(k)}$. If $w_{k}^{(1)}$ and $w_{k}^{(2)}$ are parallel for some $k$, then the coefficient is equal to 0 . So we assume that they are not parallel for all $k$, in other words, one of $w_{k}^{(1)}$ and $w_{k}^{(2)}$ is the non-vertical string. Let $1 /\left|f_{k}(t)\right|$ denote the distance of the end points of $\Omega^{(k)}$ at level $t$, i.e. $f_{k}(t)= \pm 1 /\left(t-a_{k}\right)$ for some $a_{k} \in \mathbf{R}$. We choose the signature of $f(t)$ as follows. $f_{k}(t)>0$ if the distance is increasing with respect to $t$ and the orientations of $w_{1}, w_{2}$ are coherent, or the distance is decreasing and the orientation of $w_{1}, w_{2}$ are not coherent; $f_{1}(t)= \pm 1 /(t-a)$. By repeating similar computation to get (3.5), we know that the coefficient is

$$
\frac{1}{(2 \pi i)^{p+r}} \int_{\left[\varepsilon, t_{1}\right]}^{(r)} \frac{1}{p !}\left(\log t_{1}\right)^{p} f_{*}\left(s_{*}\right) d s_{*} \Omega^{(r)} \ldots \Omega^{(1)} \omega^{p} .
$$

The limit of the above integral is finite because $f_{1}(t)= \pm 1 /\left(t-a_{1}\right)$ with $a_{1} \neq 0$.

Similarly, we can prove the finiteness for the maximal point case.

Due to the 4-term relation, we have

Lemma 5.3. Let $T$ be a tangle and $t_{1}<t_{2}<\cdots<t_{m} \in \mathbf{R}$ such that $T$ has $a$ maximal or minimal point at level $t=t_{i}$. Let $T^{\prime}$ be a tangle obtained from $T$ by a horizontal move keeping each point of levels $t=t_{i}$ invariant. Then $Z_{f}\left(T^{\prime}\right)=$ $Z_{f}(T)$, where $Z_{f}$ is defined in Proposition 5.1.

Lemma 5.4. Let $T$ be a tangle and $t_{1}<t_{2}<\cdots<t_{m} \in \mathbf{R}$ such that $T$ has a maximal or minimal point at level $t=t_{l}$. Let $T(\varepsilon)$ be a tangle equal to $T$ except a neighborhood of a maximal or minimal point at level $t_{i}$ of $T$ where $T(\varepsilon)$ is given as in Fig. 14. This figure explains the case of maximal point. Assume that the two strings used for moving the extremal point are parallel to each other, they are not parallel to $\mathbf{C}$ and they are contained in a plane transversal to $\mathbf{C} . W e$ also assume that the level of the moved maximal (resp. minimal) point is less than $t_{i+1}$ (resp. more than $\left.t_{i-1}\right)$. Here we use conventions $t_{0}=-\infty$ and $t_{m+1}=\infty$. Then $Z_{f}(T(\varepsilon))=Z_{f}(T)$. 

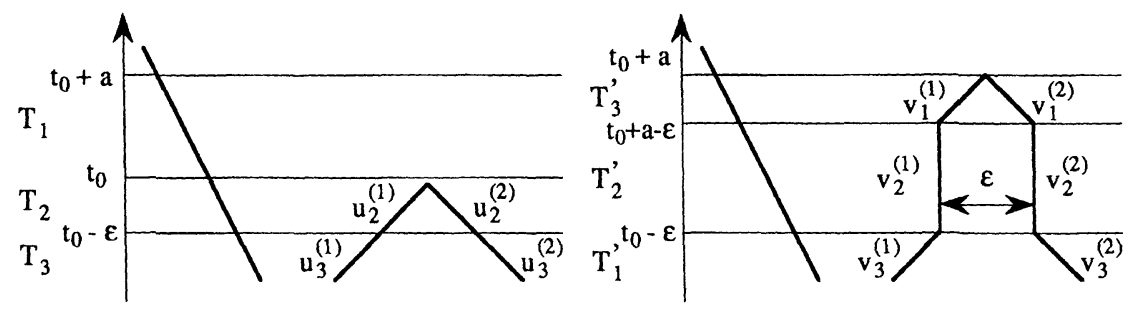

Fig. 14. Vertical move of a maximal point

Proof. We prove for the maximal point case since proof for a minimal point is similar. We split $T$ to three parts $T_{1}, T_{2}$ and $T_{3}$, and split $T^{\prime}$ to three parts $T_{1}^{\prime}, T_{2}^{\prime}$ and $T_{3}^{\prime}$. Let $u_{2}^{(1)}, u_{2}^{(2)}, u_{3}^{(1)}$ and $u_{3}^{(2)}$ be parts of a Wilson loop of $T$ and let $v_{1}^{(1)}, v_{1}^{(2)}, v_{2}^{(1)}, v_{2}^{(2)}, v_{3}^{(1)}$ and $v_{3}^{(2)}$ be parts of a Wilson loop as in Fig. 14. A configuration for $T_{i}$ is called type $I$ if it has only chords with end points on $u_{i}^{(1)}$ and $u_{i}^{(2)}$. A configuration for $T_{l}$ is called type $I I$ if it has a chord with one end point on $u_{l}^{(1)}$ or $u_{l}^{(2)}$ and another end point on neither $u_{i}^{(1)}$ nor $u_{l}^{(2)}$. A configuration for $T_{i}$ is called type $I I I$ if it has only chords with neither end points on $u_{i}^{(1)}$ nor $u_{l}^{(2)}$. We also define types of configurations for $T_{i}^{\prime}$ similarly. Let $Z\left(T_{i}\right)=A_{l}^{I}+A_{l}^{I I}+A_{i}^{I I I}$, where $A_{l}^{j}$ denote the integral for configurations of type $j$. Similarly, $Z\left(T_{i}^{\prime}\right)=B_{l}^{I}+B_{l}^{I I}+B_{i}^{I I I}$.

We have $Z(T)=Z\left(T_{1}\right) Z\left(T_{2}\right) Z\left(T_{3}\right)=A_{1}^{I I I}\left(A_{2}^{I}+A_{2}^{I I}+A_{2}^{I I I}\right)\left(A_{3}^{I}+A_{3}^{I I}+A_{3}^{I I I}\right)$ and $Z\left(T^{\prime}\right)=Z\left(T_{1}^{\prime}\right) Z\left(T_{2}^{\prime}\right) Z\left(T_{3}^{\prime}\right)=\left(B_{1}^{I}+B_{1}^{I I}+B_{1}^{I I I}\right)\left(B_{2}^{I I}+B_{2}^{I I I}\right)\left(B_{3}^{I}+B_{3}^{I I}+B_{3}^{I I I}\right)$. Note that $B_{2}^{I}=0$. By using $\lim _{\varepsilon \rightarrow 0} \varepsilon^{k}(\log \varepsilon)^{\ell}=0$ for $k>0$, we have $Z(T)=$ $\lim _{\varepsilon \rightarrow 0} Z(T)=\lim _{\varepsilon \rightarrow 0} A_{1}^{I I I}\left(A_{2}^{I}+A_{2}^{I I}+A_{2}^{I I I}\right)\left(A_{3}^{I}+A_{3}^{I I}+A_{3}^{I I I}\right)=\lim _{\varepsilon \rightarrow 0} A_{1}^{I I I} A_{2}^{I}\left(A_{3}^{I}\right.$ $\left.+A_{3}^{I I}+A_{3}^{I I I}\right)$ and $Z\left(T^{\prime}\right)=\lim _{\varepsilon \rightarrow 0} Z\left(T^{\prime}\right)=\lim _{\varepsilon \rightarrow 0}\left(B_{1}^{I}+B_{1}^{I I}+B_{1}^{I I I}\right)\left(B_{2}^{I I}+B_{2}^{I I I}\right)$ $\left(B_{3}^{I}+B_{3}^{I I}+B_{3}^{I I I}\right)=\lim _{\varepsilon \rightarrow 0} B_{1}^{I}\left(B_{2}^{I I}+B_{2}^{I I I}\right)\left(B_{3}^{I}+B_{3}^{I I}+B_{3}^{I I I}\right)$. Here we use the invariance of $Z_{f}$ under the horizontal move and use the condition for the level of new maximal point. We also have $\lim _{\varepsilon \rightarrow 0} B_{1}^{I} B_{2}^{I I}=0$, and $Z\left(T^{\prime}\right)=\lim _{\varepsilon \rightarrow 0} B_{1}^{I} B_{2}^{I I I}\left(B_{3}^{I}+\right.$ $\left.B_{3}^{I I}+B_{3}^{I I I}\right)$. Noting that $B_{1}^{I}=A_{2}^{I}, B_{2}^{I I I}=A_{1}^{I I I}$ and $B_{1}^{I} B_{2}^{I I I}=B_{2}^{I I I} B_{1}^{I}$, we get $Z(T)=$ $Z\left(T^{\prime}\right)$.

An isotopy of $\mathbf{R}^{3}=\mathbf{R} \times \mathbf{C}$ is called horizontal if it preserves the first $(\mathbf{R})$ component of each point. By using previous two lemmas, we get the following.

Lemma 5.5. Let $T^{\prime}$ be a tangle obtained from $T$ by a horizontal isotopy. Then $Z_{f}\left(T^{\prime}\right)=Z_{f}(T)$.

By using this lemma, we can remove the condition for the new maximal or minimal point in the lemma for a vertical move.

Lemma 5.6. Let $T(\varepsilon)$ be a tangle equal to $T$ except a neighborhood of a maximal or minimal point where $T(\varepsilon)$ is given as in Fig. 14. This figure explains the case of maximal point. Assume that the two strings $v_{2}^{(1)}$ and $v_{2}^{(2)}$ are parallel to each other, they are not parallel to $\mathbf{C}$ and they are contained in a plane transversal to C. Then $Z_{f}(T(\varepsilon))=Z_{f}(T)$.

Due to the above two lemmas, $Z_{f}(T)$ is invariant for Reidemeister moves except the stretching moves. As in the case of original Kontsevich's integral, we normalize $Z_{f}(T)$ so that it is equal for tangles $T T^{\prime}$ in Fig. 7. Let 


$$
\gamma_{f}=Z_{f}(U),
$$

where $U$ is the diagram in Fig. 5. Let $T$ be a tangle with $k$ numbered component and

$$
\hat{Z}_{f}(T)=\left(\gamma_{f}^{-m_{1}} \otimes \ldots \otimes \gamma_{f}^{-m_{k}}\right) \cdot Z_{f}(T),
$$

where $m_{j}$ is the number of maximal points of the $j^{\text {th }}$ component of $T$ and so $\gamma_{f}^{-m_{J}}$ acts on the $j^{\text {th }}$ component.

Theorem 5.9. $\hat{Z}_{f}(T)$ is an invariant of regular isotopy.

Proof is similar to $\hat{Z}$ case.

Remark. 5.10. $\hat{Z}_{f}(T)$ is not invariant by the twisting at the minimal and the maximal points. Let $T$ be a tangle and let $T^{\prime}$ be a tangle twisted by angle $\pm \pi$ at a maximal (resp. minimal) point of $T$. Then $\hat{Z}_{f}\left(T^{\prime}\right)=e^{ \pm \Omega / 2} \hat{Z}_{f}(T)\left(\right.$ resp. $\left.e^{\mp \Omega / 2} \hat{Z}_{f}(T)\right)$.

Now we compute the values of $\widehat{Z}_{f}(T)$ for simple q-tangles in Proposition 0.3, which are given in Theorem 0.4.

Proof of Theorem 0.4. If the tangle $T$ is $R_{k}, R_{k}^{-1}$ or $I$, the argument for Lemmas 2.7, 2.10 and Theorem 3.22 is also good for $\widehat{Z}$ and so we get the values of these cases as in Theorem 0.4. For $T=E_{k}^{*}$, we show that

$$
\lim _{\varepsilon \rightarrow 0} \varepsilon_{\alpha, r} Z_{f}(T, \alpha, \beta) \varepsilon_{\beta, s}=E_{k}^{*} .
$$

We may assume that the tangle $E_{k}^{*}$ is of the form as in Fig. 13 and the string with the cusp connect the $k^{\text {th }}$ and $k+1^{\text {th }}$ points at the top. It is enough to show that $\lim _{t_{1} \rightarrow 0} t_{1} \Omega_{k k+1} /(2 \pi i) Z_{f}\left(T_{0}^{t_{1}}, \alpha, \beta\right)=E_{k}^{*}$. For a configuration containing a cord other than $\Omega_{k k+1}$, the coefficient of the integral $Z_{f}\left(T_{0}^{t_{1}}, \alpha, \beta\right)$ of this configuration is bounded by $O\left(t_{1}\left(\log t_{1}\right)^{\ell}\right)$ for some $\ell$ from the proof of Proposition 5.1. Hence, for a configuration containing a chord other than $\Omega_{k k+1}$, the coefficient

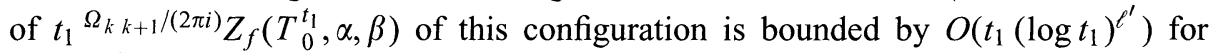
some $\ell^{\prime}$. Therefore, the limit of this coefficient is equal to 0 . The remaining configurations are $\Omega_{k k+1}{ }^{d}$ for nonnegative integers $d$. The coefficient of $\Omega_{k k+1}{ }^{d}$ is $\frac{1}{(2 \pi i)^{d}} \sum_{k+\ell=d} \frac{(-1)^{\prime}}{k ! \ell !}\left(\log t_{1}\right)^{k+\ell}$, which is equal to 1 if $d=0$ and 0 if otherwise. This implies (5.11). The proof for the tangle $E_{k}$ is similar. $E_{k}$ contains a maximal point, so we have to multiply the factor $\gamma_{f}$.

For a q-tangle $(T, \sigma, \tau)$, we have another invariant $\widehat{Z}_{0}(T) \in \mathscr{A}(T)$ constructed from $\widehat{Z}(T, \sigma, \tau) \in \mathscr{A}_{0}(T)$ as follows. Let $\psi$ be a mapping from $\mathscr{A}(T)$ to $\mathscr{A}(T)$ defined by

$$
\psi(\Omega)=\Omega-1 / 2\left(\delta_{1}+\delta_{2}\right)
$$

for a cord $\Omega$, where $\delta_{1}$ (resp. $\delta_{2}$ ) be a cord connecting two points in a small neighborhood of one (resp. another) end point of $\Omega . \psi$ is factored by $\mathscr{A}_{0}(T)$ and let $\psi_{0}$ be the factored mapping from $\mathscr{A}_{0}(T)$ to $\mathscr{A}(T)$. Let $\widehat{Z}_{0}(T, \sigma, \tau)=\psi_{0}(\widehat{Z}(T, \sigma, \tau))$. Then $\widehat{Z}_{0}$ is also an ambient isotopy invariant of q-tangles, while $\widehat{Z}_{f}$ is regular isotopy invariant of framed links. We have following relation between $\widehat{Z}_{f}$ and $\widehat{Z}_{0}$. 
Theorem 5.13. For a q-tangle $(T, \sigma, \tau)$,

$$
\widehat{Z}_{f}(T, \sigma, \tau)=\left(e^{\left(w_{1}+\sum_{1 \neq 1} \ell_{1}\right) \Theta / 2} \otimes \ldots \otimes e^{\left(w_{h}+\sum_{1 \neq h} l_{h}\right) \Theta / 2}\right) \cdot \widehat{Z}_{0}(T, \sigma, \tau),
$$

where $k$ is the number of components of $T, w$, is the writhe of the knot of the $j^{\text {th }}$ component, $\ell_{p q}$ is the linking number of the $p^{\text {th }}$ and $q^{\text {th }}$ component, $\Theta$ is the chord diagram on a circle with one chord, and $\Theta^{i}$ in the Taylor series of the exponentials is the product of $j$ copies of $\Theta$ in $\mathscr{A}$.

Proof. Due to Proposition 0.3, it is enough to prove for q-tangles in Proposition 0.3. We have $\psi\left(Z_{f}(T)\right)=\psi(Z(T))=Z_{0}(T)$ since $\psi\left(E \varepsilon^{\Omega}\right)\left(\right.$ resp. $\left.\psi\left(\varepsilon^{-\Omega} E^{*}\right)\right)$ is equal to $E$ (resp. $\left.E^{*}\right)$. We compare $\widehat{Z}_{f}(T, \sigma, \tau)$ and $\psi\left(\widehat{Z}_{f}(T, \sigma, \tau)\right)$. We first compare $Z_{f}(T, \sigma, \tau)$ and $\psi\left(Z_{f}(T, \sigma, \tau)\right)$, then show that $\gamma_{f}=\gamma$. For $T=E_{k}$ or $E_{k}^{*}$, $\psi\left(Z_{f}(T, \alpha, \beta)\right)=Z(T, \alpha, \beta)$, since $\widehat{Z}_{f}(T, \alpha, \beta)=E_{k}$ or $E_{k}^{*}$. For $T=R_{k}$ or $R_{k}^{-1}$, we have $Z_{f}(T, \alpha, \alpha)=e^{ \pm \Omega / 2}$ and so $\psi\left(Z_{f}(R)\right)=e^{ \pm\left(\Omega / 2-\delta_{1} / 4-\delta_{2} / 4\right)}$. We show

$$
\psi\left(Z_{f}(I, \eta(k k+1), \eta)\right)=Z_{f}(I, \eta(k k+1), \eta)
$$

for a trivial tangle $I$. Recall that $Z_{f}(I,(12),(21))=\phi\left(\Omega_{12}, \Omega_{23}\right)$. We first show

$$
\psi\left(\phi\left(\Omega_{12}, \Omega_{23}\right)\right)=\phi\left(\Omega_{12}, \Omega_{23}\right),
$$

where $I$ is the trivial tangle with three strings. Let $\delta_{l}^{k},(i=1,2,3)$ be $k$ small cords connecting $2 k$ points of a small neighborhood of a point of the $i^{\text {th }}$ string without crossings. Let $\delta_{k \ell}=\frac{1}{2}\left(\delta_{k}+\delta_{t}\right)$. Let $\Psi_{1}$ be a mapping acting on $\mathscr{A}(I)$ defined by $\Psi_{1}\left(\Omega_{k_{1} k_{f}^{\prime}} \ldots \Omega_{k_{2} k_{2}^{\prime}} \Omega_{k_{1} k_{1}^{\prime}}\right)=\sum_{r=1}^{k} \Omega_{k_{,} k_{1}^{\prime}} \ldots \Psi\left(\Omega_{k_{r} k_{r}^{\prime}}\right) \ldots \Omega_{k_{1} k_{1}^{\prime}}$ and $\Psi\left(\Omega_{k_{1} k_{r}^{\prime}}\right)=\Omega_{k_{1} k_{r}^{\prime}}$ - $\delta_{12}$ if $k_{r}=1$ and $\Omega_{k_{1} k_{l}^{\prime}}$ if otherwise. Let $\Psi_{2}$ be a mapping acting on $\mathscr{A}(I)$ defined similarly to $\Psi_{1}$, with $\Psi_{2}\left(\Omega_{k_{1} k_{1}^{\prime}}\right)=\Omega_{k_{1} k_{1}^{\prime}}-\delta_{23}$ if $k_{r}^{\prime}=3$ and $\Omega_{k_{r} k_{l}^{\prime}}$ if otherwise. Then $\psi_{0}\left(\phi\left(\Omega_{12}, \Omega_{23}\right)\right)=\exp \left(\Psi_{1}+\Psi_{2}\right) \phi\left(\Omega_{12}, \Omega_{23}\right)$. From (3.10), we have

$$
\phi\left(\Omega_{12}, \Omega_{23}\right)=\sum_{m=0}^{\infty} \sum_{g=0}^{[m / 2]} \sum_{\substack{\alpha, J)=0,|J|=m}} c_{J, 0,0} \alpha
$$

where

$$
\alpha=\sum_{\substack{(0, \ldots, 0) \leqq J^{\prime} \leqq J \\
\text { component }}}(-1)^{\left|J^{\prime}\right|} \prod_{J=1}^{g}\left(\begin{array}{c}
p_{j} \\
p_{j}^{\prime}
\end{array}\right)\left(\begin{array}{c}
q_{j} \\
q_{J}^{\prime}
\end{array}\right) \Omega_{23}{ }^{p\left(J^{\prime}\right)} \Omega_{J-J^{\prime}} \Omega_{12}{ }^{q\left(J^{\prime}\right)} .
$$

Here $J^{\prime}=\left(p_{1}^{\prime}, q_{1}^{\prime}, \ldots, p_{g}^{\prime}, q_{g}^{\prime}\right)$. We show that $\Psi_{1}$ and $\Psi_{2}$ send $\alpha$ to 0 .

We see the coefficient of $\delta_{12} \Omega_{23}{ }^{p\left(J^{\prime}\right)} \Omega_{J-J^{\prime}} \Omega_{12}{ }^{q\left(J^{\prime}\right)}$ in $\Psi_{1}(\alpha)$. This is

$$
\begin{gathered}
(-1)^{p\left(J^{\prime}\right)+1}\left(\begin{array}{c}
q_{1} \\
q_{1}^{\prime}
\end{array}\right) \cdots\left(\begin{array}{c}
q_{g} \\
q_{g}^{\prime}
\end{array}\right)\left((-1)^{p\left(J^{\prime}\right)} p\left(J^{\prime}\right) \prod_{j=1}^{g}\left(\begin{array}{c}
p_{j} \\
p_{l}^{\prime}
\end{array}\right)\left(\begin{array}{c}
q_{j} \\
q_{j}^{\prime}
\end{array}\right)\right. \\
\left.+\sum_{l=1}^{r}\left(p_{l}-p_{i}^{\prime}+1\right)\left(\begin{array}{c}
p_{1} \\
p_{1}^{\prime}
\end{array}\right) \ldots\left(\begin{array}{c}
p_{i} \\
p_{i}^{\prime}-1
\end{array}\right) \ldots\left(\begin{array}{c}
p_{r} \\
p_{r}^{\prime}
\end{array}\right)\right) .
\end{gathered}
$$


Since $\left(p_{i}-p_{i}^{\prime}+1\right)\left(\begin{array}{c}p_{l} \\ p_{i}^{\prime}-1\end{array}\right)=p_{i}^{\prime}\left(\begin{array}{c}p_{i} \\ p_{l}^{\prime}\end{array}\right),(5.18)$ is equal to 0 . Similarly, $\Psi_{2}$ sends $\alpha$ to 0 . Hence, $\psi_{0}(\alpha)=\exp \left(\Psi_{1}+\Psi_{2}\right)(\alpha)=\alpha$ and so $\psi_{0}$ does not change $\phi\left(\Omega_{12}, \Omega_{23}\right)$.

We show (5.16) for a trivial tangle $I$ with more than three strings by similar argument. We use the notations in Theorem 0.4. Since

$$
\psi\left(\sum_{\substack{a \leqq p \leqq b \\ b+1 \leqq q \leqq c}} r_{p} r_{q} \Omega_{p q}\right)=\sum_{\substack{a \leqq p \leqq b \\ b+1 \leqq q \leqq c}} r_{p} r_{q} \Omega_{p q}-\sum_{\substack{a \leqq p \leqq b \\ b+1 \leqq q \leqq c}} r_{p} r_{q}\left(\delta_{p}+\delta_{q}\right)
$$

we can use the previous argument to prove (5.16) with $\sum_{\substack{k \leqq p \leqq J_{0} \\ \text { s. }}} r_{q} r_{q} \Omega_{p q}$, $\sum_{\substack{J_{0}+1 \leqq p \leqq J_{1} \\ J_{1}+1 \leqq q \leqq 1}} r_{p} r_{q} \Omega_{p q}, \sum_{\substack{k \leqq p \leqq J_{0} \\ J_{0}+1 \leqq q \leqq J_{1}}} r_{p} r_{q}\left(\delta_{p}+\delta_{q}\right)$, and $\sum_{\substack{J_{0} \leqq p \leqq J_{1} \\ J_{1}+1 \leqq q \leqq \prime}} r_{p} r_{q}\left(\delta_{p}+\delta_{q}\right)$ instead of $\Omega_{12}, \Omega_{23}, \delta_{12}$ and $\delta_{23}$ respectively.

The above argument implies

$$
Z_{f}(T, \sigma, \tau)=\left(e^{\left(w_{1}+\sum_{j \neq 1} \ell_{1 j}\right) \Theta / 2} \otimes \ldots \otimes e^{\left(w_{k}+\sum_{j \neq k} \ell_{k J}\right) \Theta / 2}\right) \cdot \psi_{0}(Z(T, \sigma, \tau)) .
$$

In the rest of proof, we show $\psi_{0}(\gamma)=\gamma_{f}$, where $\gamma=Z(U)$ and $\gamma_{f}=Z_{f}(U)$ for the diagram $U$ in Fig. 5. We know that $\gamma_{f}=E_{2} \phi\left(-\Omega_{12},-\Omega_{23}\right) E_{1}^{*}$, and so $\psi\left(\gamma_{f}\right)=$ $\gamma_{f}$. On the other hand, $\psi^{2}=\psi$ and $\psi_{0}$ gives an isomorphism between $\mathscr{A}_{0}(T)$ and the image of $\psi$ in $\mathscr{A}(T)$ for any tangle $T$. Note that $\psi_{0}^{-1}$ is equal to the natural projection from $\psi(\mathscr{A}(T)) \subset \mathscr{A}(T)$ to $\mathscr{A}_{0}(T)$. Since $\gamma_{f} \in \psi(\mathscr{A}(I))$, where $I$ is the trivial $(1,1)$-tangle, $\psi_{0}^{-1}\left(\gamma_{f}\right)=\gamma$, and so $\psi_{0}(\gamma)=\gamma_{f}$.

Remark. . Another proof for (5.16) is in Appendix of [19].

\section{Relation to a Quasi-Hopf Algebra}

Let $r^{(k)}=\underbrace{(1, \ldots, 1)}_{k}$ and $\mathscr{M}^{(k)}=\mathscr{A}\left(I^{(k)}\right)$, where $I^{(k)}$ is the trivial tangle in $\mathscr{T}^{\left(r^{(k)}, r^{(k)}\right)}$ whose strings are oriented downwards. Then $\mathscr{M}^{(k)}$ is an algebra with the product induced by the product of tangles. We call $\mathscr{M}^{(k)}$ the chord diagram algebra of degree $k . \mathscr{M}^{(0)}=\mathbf{C}$ by definition. For a sequence of algebras $\mathbf{C}=\mathscr{M}^{(0)}, \mathscr{M}^{(1)}, \ldots, \mathscr{M}^{(k)}, \ldots$, we introduce a structure similar to a Hopf algebra structure. The algebra $\mathscr{A}$ is isomorphic to $\mathscr{M}^{(1)}$. We have an inclusion $\mathscr{A}^{\otimes k} \sim\left(\mathscr{M}^{(1)}\right)^{\otimes k} \subset \mathscr{M}^{(k)}$. We also have a linear mapping $\mathscr{M}^{(k)} \rightarrow \mathscr{A}^{\otimes k}$ defined by the following. Let $D$ be a chord diagram in $\mathscr{M}^{(k)}$. Removing all the chords of $D$ with two end points at different strings, we get a disjoint union of $k$ chord diagrams $D_{1}, \ldots, D_{k}$. Let $D_{i}^{\prime}$ be the chord diagram on a circle obtained from $D_{i}$ by connecting the ending point and the starting point of the string of $D_{l}$. Then $D_{1}^{\prime} \otimes$ $\ldots \otimes D_{k}^{\prime} \in \mathscr{A}^{\otimes k}$. The projection $\mathscr{M}^{(k)} \rightarrow \mathscr{A}^{\otimes k}$ is a linear extension of the above mapping for chord diagrams. We extend the "Hopf algebra structure" of $\mathscr{A}$ to $\mathscr{M}^{(0)}$, $\mathscr{M}^{(1)}, \ldots, \mathscr{M}^{(k)}, \ldots$ with respect to the above inclusions and projections. 
We put numbers $1, \ldots, k$ to the strings of chord diagrams in $\mathscr{M}^{(k)}$. We first extend the multiplication $m: \mathscr{A} \otimes \mathscr{A} \rightarrow \mathscr{A}$ to a linear mapping $m: \mathscr{M}^{(2)} \rightarrow \mathscr{M}^{(1)}$. For a chord diagram $D \in \mathscr{M}^{(2)}$, we can make a chord diagram $D^{\prime} \in \mathscr{M}^{(1)}$ by connecting the ending point of the first string to the starting point of the second string. Let $m$ be a linear extension of the above mapping. We sometimes need an extension of the mapping id ${ }^{\otimes(\ell-1)} \otimes m \otimes \mathrm{id}^{\otimes(k-\ell-1)}: \mathscr{A}^{\otimes k} \rightarrow \mathscr{A}^{\otimes(k-1)}$ to $\mathrm{id}^{\otimes(\ell-1)} \otimes$ $m \otimes \mathrm{id}^{\otimes(k-\ell-1)}: \mathscr{M}^{(k)} \rightarrow \mathscr{M}^{(k-1)}$. In our notation, $\mathrm{id}^{\otimes(\ell-1)} \otimes m \otimes \mathrm{id}^{\otimes(k-\ell-1)}$ does not mean the usual tensor product any longer. For a chord diagram $D \in \mathscr{M}^{(k)}$, we can make a chord diagram $D^{\prime} \in \mathscr{M}^{(k-1)}$ by connecting the ending point of the $\ell^{\text {th }}$ string to the starting point of the $(\ell+1)^{\text {th }}$ string. Let id ${ }^{\otimes(\ell-1)} \otimes m \otimes \mathrm{id}^{\otimes(k-\ell-1)}$ be a linear extension of this mapping. This extended mapping and the original one commute with the inclusions $\mathscr{A}^{\otimes k} \rightarrow \mathscr{M}^{(k)}$ and $\mathscr{A}^{\otimes(k-1)} \rightarrow \mathscr{M}^{(k-1)}$. The unit 1 is the chord diagram without any chords.

Next, we generalize the coproduct $\Delta: A \rightarrow \mathscr{A} \otimes \mathscr{A}$ to $\Delta: \mathscr{M}^{(1)} \rightarrow \mathscr{M}^{(2)}$. Let $D$ be a chord diagram in $\mathscr{M}^{(1)}$ and $p_{1}, \ldots, p_{k}$ be the points on the non-closed string of $D$ where the chords are attached. Take a duplicate of the string and let $p_{1}^{\prime}, \ldots, p_{k}^{\prime}$ be the corresponding points to $p_{1}, \ldots, p_{k}$ on the new string. Then replace chords as follows. If a chord, say $\Omega_{p_{l} p_{j}}$, connects $p_{i}$ and $p_{j}$ then replace it by $\Omega_{p_{l} p_{j}}+\Omega_{p_{l}^{\prime} p_{l}}+\Omega_{p_{l} p_{l}^{\prime}}+\Omega_{p_{l}^{\prime} p_{i}^{\prime}}$. By the above replacement, $D$ is replaced by a sum of $2^{k}$ chord diagrams in $\mathscr{M}^{(2)}$. Let $\Delta$ be the linear extension of the above mapping. We sometimes need an extension of the mapping $\mathrm{id}^{\otimes(t-1)} \otimes \Delta \otimes \mathrm{id}^{\otimes(k-t)}: \mathscr{A}^{\otimes k} \rightarrow$ $\mathscr{A}^{\otimes(k+1)}$ to $\mathrm{id}^{\otimes(\ell-1)} \otimes \Delta \otimes \mathrm{id}^{\otimes(k-\ell)}: \mathscr{M}^{(k)} \rightarrow \mathscr{M}^{(k+1)}$. In our notation, $\mathrm{id}^{\otimes(\ell-1)} \otimes$ $\Delta \otimes \mathrm{id}^{\otimes(k-\ell)}$ does not mean the usual tensor product any longer. Let $D$ be a chord diagram in $\mathscr{M}^{(k)}$ and $p_{1}, \ldots, p_{k}$ be the points on the $\ell^{\text {th }}$ string of $D$ where the chords are attached. Take a duplicate of the $\ell^{\text {th }}$ string and let $p_{1}^{\prime}, \ldots, p_{k}^{\prime}$ be the corresponding points to $p_{1}, \ldots, p_{k}$ on the new string. Then replace chords as follows. If a chord, say $\Omega_{p_{l} p_{J}}$, connect $p_{i}$ and $p_{j}$ then replace it by $\Omega_{p_{l} p_{l}}+\Omega_{p_{l}^{\prime} p_{J}}+\Omega_{p_{l} p_{l}^{\prime}}+$ $\Omega_{p_{l}^{\prime} p_{j}^{\prime}}$. If a chord, say $\Omega_{p_{l} q}$, connect the point $p_{l}$ and a point $q$ not on the $\ell^{\text {th }}$ string, then replace $\Omega_{p_{l} q}$ by $\Omega_{p_{l} q}+\Omega_{p_{l}^{\prime} q}$. By the above replacement, $D$ is replaced by a sum of $2^{k}$ chord diagrams in $\mathscr{M}^{(2)}$. Let $\mathrm{id}^{\otimes(\ell-1)} \otimes \Delta \otimes \mathrm{id}^{\otimes(k-\ell)}$ be a linear extension of this mapping. This extended mapping and the original one commute with the projections $\mathscr{M}^{(k)} \rightarrow \mathscr{A}^{\otimes k}$ and $\mathscr{M}^{(k+1)} \rightarrow \mathscr{A}^{\otimes(k+1)}$.

We have a counit $\varepsilon: \mathscr{A} \sim \mathscr{M}^{(1)} \rightarrow \mathbf{C}$. For a chord diagram $D \in \mathscr{M}^{(1)}, \varepsilon(D)=1$ if $D$ is the chord diagram without any chords and $\varepsilon(D)=0$ if otherwise. We also need an extension of the mapping id ${ }^{\otimes(\ell-1)} \otimes \varepsilon \otimes \mathrm{id}^{\otimes(k-\ell)}: \mathscr{A}^{\otimes k} \rightarrow \mathscr{A}^{\otimes(k-1)}$ to $\mathrm{id}^{\otimes(\ell-1)} \otimes \varepsilon \otimes \mathrm{id}^{\otimes(k-\ell)}: \mathscr{M}^{(k)} \rightarrow \mathscr{M}^{(k-1)}$. For a chord diagram $D \in \mathscr{M}^{(k)}, \mathrm{id}^{\otimes(\ell-1)}$ $\otimes \varepsilon \otimes \mathrm{id}^{\otimes(k-\ell)}(D)=D$ if there is no chord of $D$ ending at the $\ell^{\text {th }}$ string and $\mathrm{id}^{\otimes(\ell-1)} \otimes \varepsilon \otimes \mathrm{id}^{\otimes(k-\ell)}(D)=0$ if otherwise. Let $\mathrm{id}^{\otimes(\ell-1)} \otimes \varepsilon \otimes \mathrm{id}^{\otimes(k-\ell)}$ be a linear extension of this mapping. This extended mapping and the original counit commute with the projections $\mathscr{M}^{(k)} \rightarrow \mathscr{A}^{\otimes k}$ and $\mathscr{M}^{(k-1)} \rightarrow \mathscr{A}^{\otimes(k-1)}$.

We have an antipode $S: \mathscr{A} \rightarrow \mathscr{A}$. For a chord diagram $D \in \mathscr{M}^{(1)}, S(D)$ is a copy of $D$ with opposite orientation for the string. We also need an extension of the mapping id ${ }^{\otimes(\ell-1)} \otimes S \otimes \mathrm{id}^{\otimes(k-\ell)}: \mathscr{A}^{\otimes k} \rightarrow \mathscr{A}^{\otimes k}$ to $\mathrm{id}^{\otimes(\ell-1)} \otimes S \otimes \mathrm{id}^{\otimes(k-\ell)}$ : $\mathscr{M}^{(k)} \rightarrow \mathscr{M}^{(k)}$. Let $D$ be a chord diagram in $\mathscr{M}^{(k)}$. Change the orientation of the $\ell^{\text {th }}$ string and replace each chord $\Omega$ with just one end point at the $\ell^{\text {th }}$ string by $-\Omega$, we get a chord diagram $D^{\prime} \in \mathscr{M}^{(k)}$. Let $\mathrm{id}^{\otimes(\ell-1)} \otimes S \otimes \mathrm{id}^{\otimes(k-\ell)}$ be a linear extension of this mapping. 
Let $\phi=\psi_{f}\left(\Omega_{12}, \Omega_{23}\right) \in \mathscr{M}^{(3)}$, where $\psi_{f}\left(\Omega_{12}, \Omega_{23}\right)$ is defined in Sect. 5. Let $\mathrm{id}^{\otimes p} \otimes \phi \otimes \mathrm{id}^{\otimes q}$ be an element of $\mathscr{M}^{(p+q+3)}$ obtained by adding $p+q$ strings, numbered from 1 to $p$ and from $p+4$ to $p+q+3$. The $\ell^{\text {th }}$ string of $\psi$ is numbered by $\ell+p$. By the construction of $\phi$, we have the following relations.

$(\mathrm{id} \otimes \Delta)(\Delta(a))=(\Delta \otimes \mathrm{id})(\Delta(a))=\phi(\Delta \otimes \mathrm{id})(\Delta(a)) \phi^{-1}, \quad a \in \mathscr{M}^{(1)}$

$(\mathrm{id} \otimes \mathrm{id} \otimes \Delta) \phi(\Delta \otimes \mathrm{id} \otimes \mathrm{id}) \phi=(\mathrm{id} \otimes \phi)(\mathrm{id} \otimes \Delta \otimes \mathrm{id}) \phi(\phi \otimes \mathrm{id})$,

$(\varepsilon \otimes \mathrm{id}) \Delta=\mathrm{id}=(\mathrm{id} \otimes \varepsilon) \Delta$,

$(\mathrm{id} \otimes \varepsilon \otimes \mathrm{id}) \phi=1$.

These correspond to relations for a quasi-bialgebra, and so the sequence $M^{(0)}, M^{(1)}$, $\ldots, M^{(k)}, \ldots$ is a generalization of a quasi-Hopf algebra in some sense.

Let $\gamma_{f}=Z_{f}(U)$. Let $\imath: \mathbf{C} \rightarrow \mathscr{M}^{(1)}$ be a linear extension of the unit 1 and $\mathrm{id}^{\otimes(\ell-1)} \otimes \imath \otimes \mathrm{id}^{\otimes(k-\ell)}: M^{(k)} \rightarrow \mathscr{M}^{(k+1)}$ be the linear mapping defined by the following. Let $D$ be a chord diagram in $M^{(k)}$. Adding a string to $D$, we get a chord diagram $D^{\prime}$. We put a number $p$ (resp. $p+1$ ) to the strings of $D^{\prime}$ corresponding to the $p^{\text {th }}$ string of $D$ if $p<\ell$ (resp. $p \geqq \ell$ ) and put $\ell$ to the newly added string. Then $\mathrm{id}^{\otimes(\ell-1)} \otimes \imath \otimes \mathrm{id}^{\otimes(k-\ell)}(D)=D^{\prime}$. From the definition of $m, \Delta, \varepsilon$ and $S$, we have

$$
\begin{aligned}
\left(\mathrm{id}^{\otimes(\ell-1)} \otimes m \otimes \mathrm{id}^{\otimes(k-\ell)}\right)\left(\mathrm{id}^{\otimes(\ell-1)} \otimes S \otimes \mathrm{id}^{\otimes(k-\ell+1)}\right)\left(\mathrm{id}^{\otimes(\ell-1)} \otimes \Delta \mathrm{id}^{\otimes(k-\ell)}\right) \cdot a \\
=\left(\mathrm{id}{ }^{\otimes(\ell-1)} \otimes l \otimes \mathrm{id}^{\otimes(k-\ell)}\right)\left(\mathrm{id}^{\otimes(\ell-1)} \otimes \varepsilon \otimes \mathrm{id}^{\otimes(k-\ell)}\right) \cdot a, \\
\left(\mathrm{id}^{\otimes(\ell-1)} \otimes m \otimes \mathrm{id}^{\otimes(k-\ell)}\right)\left(\mathrm{id}^{\otimes(\ell)} \otimes S \otimes \mathrm{id}^{\otimes(k-\ell)}\right)\left(\mathrm{id}^{\otimes(\ell-1)} \otimes \Delta \mathrm{id}^{\otimes(k-\ell)}\right) \cdot a \\
=\left(\mathrm{id}^{\otimes(\ell-1)} \otimes l \otimes \mathrm{id}^{\otimes(k-\ell)}\right)\left(\mathrm{id}^{\otimes(\ell-1)} \otimes \varepsilon \otimes \mathrm{id}^{\otimes(k-\ell)}\right) \cdot a,
\end{aligned}
$$

for $a \in \mathscr{M}^{(k)}$ and

$$
\begin{aligned}
& m(m \otimes \mathrm{id})(\mathrm{id} \otimes S \otimes \mathrm{id}) \phi=\gamma_{f}, \\
& m(m \otimes \mathrm{id})(S \otimes \mathrm{id} \otimes S) \phi^{-1}=\gamma_{f} .
\end{aligned}
$$

These correspond to relations for a quasi-Hopf algebra, and so the sequence $M^{(0)}, M^{(1)}, \ldots, M^{(k)}, \ldots$ is a generalization of a quasi-Hopf algebra in some sense.

Let $R=e^{\Omega_{12} / 2} \in \mathscr{M}^{(2)}$. Then we have

$$
\begin{aligned}
\Delta(a) & =R \Delta(a) R^{-1}, \quad a \in \mathscr{M}^{(1)}, \\
(\Delta \otimes \mathrm{id}) R & =\phi_{312} R_{13} \phi_{132}^{-1} R_{23} \phi, \\
(\mathrm{id} \otimes \Delta) R & =\phi_{231}^{-1} R_{13} \phi_{213} R_{23} \phi,
\end{aligned}
$$

where $R_{i j}=e^{\Omega_{i j} / 2}, \phi_{i j k}=\psi\left(\Omega_{i j}, \Omega_{j k}\right)$. These are relations for quasitriangularity, and so the sequence $M^{(0)}, M^{(1)}, \ldots, M^{(k)}, \ldots$ is a generalization of a quasitriangular quasi-Hopf algebra in some sense.

As the above argument shows, the sequence $M^{(0)}, M^{(1)}, \ldots, M^{(k)}, \ldots$ possess all properties needed for a quasitriangular quasi-Hopf algebra. This sequence plays the role of a quasi-triangular quasi-Hopf algebra in the construction of link invariants 
as in [2]. The 4-term relation corresponds to the classical Yang-Baxter equation. So, once we are given a solution of the classical Yang-Baxter equation, we can construct a "state model" of the sequence $M^{(0)}, M^{(1)}, \ldots, M^{(k)}, \ldots$ as in $[5,18]$, from which we get a $\mathbf{C}$-valued invariant of links. The sequence $M^{(0)}, M^{(1)}, \ldots, M^{(k)}, \ldots$ does not include the quantum groups, but is a universal object for the algebras generated by classical $r$-matrices.

Acknowledgement. We would like to express our thanks to M. Kontsevich and D. Zagier for useful discussions, and we are grateful to the Max-Planck-Institut für Mathematik for their kind hospitality.

\section{References}

1. Akutsu, Y., Deguchi, T., Wadati, M.: Exactly solvable models and new link polynomials III. J. Phys. Soc. of Japan 57, 757-776 (1988)

2. Altschuler, D., Coste, A.: Quasi-quantum goups, knots, three-manifolds, and topological field theory. Commun. Math. Phys. 150, 83-107 (1992)

3. Altschuler, D., Freidel, L.: On universal Vassiliev invariants, preprint, ENSLAPP-L 455/94, February 1994

4. Arnold, V.I.: The First European Congress of Mathematics, Paris July 1992. Basel: Birkhauser, 1993

5. Dror Bar-Natan,: On the Vassiliev knot invariants. Harvard preprint, August 1992

6. Dror Bar-Natan,: Non-Associative tangles. Harvard preprint, December 1993

7. Birman, J.S., Lin, X.S.: Knot polynomials and Vassiliev's invariants. Invent. Math. 111, 225-270 (1993)

8. Drinfel'd, V.G.: On Quasi-Hopf algebras. Leningrad Math. J. 1, 1419-1457 (1990)

9. Drinfel'd, V.G.: On quasitriangular quasi-Hopf algebras and a group closely connected with $\operatorname{Gal}(\overline{\mathbb{Q}} / \mathbb{Q})$. Leningrad Math. J. 2, 829-860 (1990)

10. Goodman, F.M., de la Harpe, P., Jones, V.F.R.: Coxeter graphs and Towers of Algebras. New York: Springer Berlin, Heidelberg, 1989

11. Hoefsmit, P.N.: Representations of Hecke algebras of finite groups with BN-paris of classical type. Thesis, the University of British Columbia

12. Kauffman, L.H.: State models and the Jones polynomial. Topology 26, 395-407 (1987)

13. Kirby, R., Melvin, P.: The 3-manifold invariants of Witten and Reshetikhin-Turaev for sl(2, C). Invent. Math. 105, 473-545 (1991)

14. Kobayashi, T., Murakami, H., Murakami, J.: Cyclotomic invariants for links. Proc. Japan Acad. Ser. A 64, 235-238 (1988)

15. Kohno, T.: Hecke algebra representations of braid groups and classical Yang-Baxter equations. In: Conformal field theory and solvable lattice models (Kyoto, 1986), Adv. Stud. Pure Math. 16, pp. 255-269

16. Kontsevich, M.: Vassiliev's knot invariants. Max-Planck-Institut für Mathematik, Bonn, preprint

17. Kuperberg, G.: The quantum $\mathrm{G}_{2}$ link invariant. Univ. of California at Berkeley, preprint August 1990

18. Le, T.Q.T., Murakami, J.: Kontsevich integral for the Homfly polynomial and relations of the multiple zeta values. to appear in Top Appl.

19. Le, T.Q.T., Murakami, J.: Kontsevich integral for the Kauffman polynomial. Max-PlanckInstitut für Mathematik, Bonn preprint

20. Murakami, J.: The parallel version of polynomial invariants of links. Osaka J. Math. 26, 1-55 (1989)

21. Reshetikhin, N.Yu.: Quasitriangular Hopf algebras and invariants of tangles. Leningrad J. Math. 1, 491-513 (1990)

22. Reshetikhin, N.Yu., Turaev, T.G.: Ribbon graphs and their invariants derived from quantum groups. Commun Math. Phys. 127, 262-288 (1990) 
23. Turaev, T.G.: The Yang-Baxter equation and invariants of links. Invent. Math. 92, 527-553 (1988)

24. Turaev, T.G.: Operator invariants of tangles and R-Matrices. Math. USSR Izvestiya 35, 411-444 (1990)

25. Vassiliev, V.A.: Cohomology of knot spaces. In: Theory of Singularities and Its Applications. V.I. Arnold (ed.) Advances in Soviet Mathematics, AMS 1, 23-69 (1990)

Communicated by H. Araki 